ARTICLE

\title{
The anaphase promoting complex impacts repair choice by protecting ubiquitin signalling at DNA damage sites
}

Kyungsoo Ha ${ }^{1,2,3, \star}$, Chengxian Ma ${ }^{4, \star}$, Han Lin ${ }^{3}$, Lichun Tang ${ }^{1,2}$, Zhusheng Lian ${ }^{5}$, Fang Zhao ${ }^{6}$, Ju-Mei Li ${ }^{7}$, Bei Zhen ${ }^{1,2}$, Huadong Pei ${ }^{1,2}$, Suxia Han ${ }^{5}$, Marcos Malumbres ${ }^{8}$, Jianping Jin ${ }^{7}$, Huan Chen ${ }^{1,2}$, Yongxiang Zhao ${ }^{6}$, Qing Zhu ${ }^{4} \&$ Pumin Zhang ${ }^{1,2,3}$

Double-strand breaks (DSBs) are repaired through two major pathways, homology-directed recombination (HDR) and non-homologous end joining (NHEJ). While HDR can only occur in $\mathrm{S} / \mathrm{G} 2$, NHEJ can happen in all cell cycle phases (except mitosis). How then is the repair choice made in $\mathrm{S} / \mathrm{G} 2$ cells? Here we provide evidence demonstrating that APCCdh1 plays a critical role in choosing the repair pathways in $S / G 2$ cells. Our results suggest that the default for all DSBs is to recruit 53BP1 and RIF1. BRCA1 is blocked from being recruited to broken ends because its recruitment signal, K63-linked poly-ubiquitin chains on histones, is actively destroyed by the deubiquitinating enzyme USP1. We show that the removal of USP1 depends on APC ${ }^{C d h 1}$ and requires Chk1 activation known to be catalysed by SSDNA-RPA-ATR signalling at the ends designated for HDR, linking the status of end processing to RIF1 or BRCA1 recruitment.

\footnotetext{
${ }^{1}$ National Center for Protein Sciences Beijing, Life Sciences Park, Beijing 102206, China. ${ }^{2}$ State Key Laboratory of Proteomics, Beijing Proteome Research Center, Beijing Institute of Radiation Medicine, 27 Taiping Road, Beijing 100850, China. ${ }^{3}$ Department of Molecular Physiology and Biophysics, Baylor College of Medicine, Houston, Texas 77030, USA. ${ }^{4}$ Department of Medical Oncology, The Second Affiliated Hospital of Xi'an Jiaotong University Medical College, Xi'an, Shaanxi 710004, China. ${ }^{5}$ Department of Radiation Oncology, The First Affiliated Hospital of Xi'an Jiaotong University Medical College, Xi'an, Shaanxi 710061, China. ${ }^{6}$ National Center for International Research of Biological Targeting Diagnosis and Therapy, and Collaborative Innovation Center for Targeting Tumor Diagnosis and Therapy, Guangxi Medical University, Guangxi 530021, China. ${ }^{7}$ Department of Biochemistry and Molecular Biology, University of Texas Health Sciences Center, Houston, Texas 77030, USA. ${ }^{8}$ Spanish National Cancer Research Centre (CNIO), Melchor Fernández Almagro 3, Madrid, Spain.

* These authors contributed equally to this work. Correspondence and requests for materials should be addressed to Y.Z. (email: yongxiang_zhao@126.com) or to Q.Z. (email: newzhuqing1972@yahoo.com) or to P.Z. (email: pzhang@bcm.edu).
} 
G enome stability is constantly challenged by DNA damage resulted from DNA replication errors and attacks by internal and external agents. Among many forms of DNA damage, double-strand breaks (DSBs) are the most dangerous to the integrity of the genome. DSBs are repaired through two pathways, homology-directed recombination (HDR) and nonhomologous end joining $(\mathrm{NHEJ})^{1-3}$. The choice between these two pathways is largely influenced by cell cycle phases, with NHEJ primarily occurring in G1 and HDR in S/G2 when homologous sequences are available from sister chromosomes ${ }^{3,4}$.

The anaphase-promoting complex/cyclosome (APC/C) is an E3 ubiquitin ligase critical for mitotic progression ${ }^{5}$. It utilizes two adaptor proteins, Cdc20 and Cdh1 (Fzr1), to bring in substrates for ubiquitination (K11-linked) ${ }^{6}$. Cdc20 functions primarily in mitosis whereas Cdh1 functions in other phases of the cell cycle, especially in G1 to prevent precocious S phase entry ${ }^{7}$. Besides the function in cell cycle regulation, APC ${ }^{C d h} 1$ has been implicated in DNA damage response. It was reported to mediate ubiquitination and degradation of USP1 to allow NER repair of UV-induced DNA damage ${ }^{8}, \operatorname{Rad} 17$ for checkpoint termination at the end of UV-induced DNA damage response ${ }^{9}$ and Plk1 to prevent precocious mitotic entry ${ }^{10}$. More recently, APC ${ }^{C d h 1}$ was proposed to regulate the clearance of CtIP, an essential HR protein, at a late time of HDR repair to prevent excessive end resection for optimal HR efficiency ${ }^{11}$.

The binding of Cdhl to APC is regulated by phosphorylation of Cdh1. From mitotic exit to sometime before the restriction point in G1, Cdh1 remains in a hypo-phosphorylated (thus active form $)^{12,13}$. During this time window, its substrates including USP1 and CtIP are degraded. However, it is phosphorylated by CDKs afterwards and becomes inactive. In budding yeast, it is the Cdc14 phosphatase that dephosphorylates (and activates) Cdh1 among many proteins phosphorylated by CDK1 upon mitotic entry 14,15 . There are two mammalian homologues of Cdc14, namely Cdc14A and B. Like Cdc14, Cdc14B is a nucleolar protein, but Cdc14A's localization remains elusive, although it was initially reported to localize to and regulate the function of centrosomes ${ }^{16,17}$. In contrast to budding yeast, neither Cdc14A nor B is required for mitotic exit ${ }^{18,19}$. Instead, it is another phosphatase, PP2A-B55 $\alpha$, that promotes mitotic exit in mammalian cells ${ }^{20}$. It remains unclear which phosphatase dephosphorylates Cdh1 during mitotic exit or in other phases of the cell cycle. However, accumulating evidence suggest that in response to DNA damage it is Cdc14B that activates Cdh1 (refs 10,21). DNA damage induces Cdc14B translocation from nucleolus to the nucleoplasm ${ }^{10}$, and it has been shown that the translocation is Chk1-dependent ${ }^{22}$. More recently, we showed that $\mathrm{Cdc} 14 \mathrm{~A}$ and B functioned redundantly in both HR- and NHEJ-mediated DNA damage repair, likely through dephosphorylating Cdh1 (ref. 21).

Dynamic ubiquitination and de-ubiquitination is known to be important in transmitting DNA damage signals and in regulating various steps in repair ${ }^{23}$. Upon DNA DSB, ATM is activated and initiates a series of phosphorylation events that ultimately result in the recruitment of two E3 ubiquitin liagases, RNF8 first and then RNF168 (ref. 24). RNF168 catalyses the formation of K63linked poly-Ub chains on $\mathrm{H} 2 \mathrm{~A} / \mathrm{H} 2 \mathrm{~B}$ which then signal BRCA1 (mainly its A-complex) recruitment ${ }^{25}$; and RNF168 also contributes to the recruitment of 53BP1 through helping the exposure of H4K20me2 (refs 26,27). BRCA1 promotes homologous recombination by further recruiting BRCA2, RAD51, and so on (refs 28,29), while 53BP1 promotes NHEJ by recruiting RIF1 (refs 30,31). RIF1 pushes for NHEJ repair by preventing BRCA1 recruitment via unknown mechanisms ${ }^{30,32}$. On the other hand, BRCA1 can also prevent RIF1 recruitment through CtIP ${ }^{31}$ and/or UHRF1 (ref. 33). Thus, RIF1 and BRCA1 expel each other from damage sites and which protein prevails is influenced by the cell cycle stage ${ }^{31}$. In G1, NHEJ dominates probably because CtIP is at a low level and is not phosphorylated, resulting in BRCA1 unable to expel RIF1, but from $S$ phase onwards, BRCA1 gains the ability to expel RIF1 and promote HR as CtIP and UHRF1 becomes phosphorylated by $\mathrm{CDK}^{31,33}$. However, despite this antagonizing relationship between BRCA1 and RIF1, RIF1 IRIF (irradiation-induced foci) and BRCA1 IRIF co-exist in S/G2 cells, and in fact, S/G2 cells can also perform NHEJ repair ${ }^{34-37}$. What then decides which DSB gets repaired through HDR and which through NHEJ in an S/G2 cell? Here we provide evidence demonstrating that APC Cdhl plays a critical role in choosing the repair pathways in S/G2. Our results suggest that the default for all DSBs in S/G2 is to recruit 53BP1 and RIF1, but not BRCA1. BRCA1 is blocked because its recruiting signal, K63linked poly-ubiquitin chains on histones, is actively destroyed by the deubiquitinating enzyme USP1. USP1 is recruited to damage sites in an MDC1-dependent manner. We show that the removal of USP1 depends on APC ${ }^{\text {Cdh } 1}$ and the activation of this E3 ubiquitin ligase is linked to end processing through Chk1 activation.

\section{Results}

RIF1 and BRCA1 IRIF co-exist in S/G2 cells. In S/G2 cells, both HDR and NHEJ can occur ${ }^{34,35}$. Indeed, RIF1 (a marker for NHEJ) and BRCA1 (a marker for HDR) foci co-exist but do not co-localize in S/G2 cells (Fig. 1a), consistent with the notion that BRCA1 and RIF1 antagonize each other ${ }^{30,31}$. We refer RIF1 foci as Pro-NHEJ and BRCA1 foci as Pro-HDR thereafter. As expected, all RIF1 IRIF colocalize with that of DNA-PKcs (Supplementary Fig. 1a). It was reported previously 30,31 that depletion of BRCA1 results in an increase in the number of RIF1 foci in S/G2 cells (Fig. 1b and Supplementary Fig. 1b). In other words, if a DSB were not occupied by BRCA1, RIF1 would take the place, suggesting that there is nothing at a Pro-HDR DSB, except BRCA1, that can prevent RIF1 from being recruited. Consistent with that, 53BP1, a factor required for RIF1 recruitment $^{30,31}$, co-localizes with BRCA1 as well as RAD51 in some but not all IRIF (irradiation induced foci) (Fig. 1c and Supplementary Fig. 1c). On the other hand, the reverse is not true. The depletion of RIF1 did not affect BRCA1 focus number in S/G2 cells (Fig. 1d), suggesting that Pro-NHEJ DSBs are not competent to recruit BRCA1. However, as reported previously ${ }^{31}$, depletion of RIF1 did allow BRCA1 focus formation in G1 (Fig. 1d), indicating that the Pro-NHEJ sites in G1 are different than those in S/G2.

RIF1 IRIF in S/G2 cells lack poly-Ub modification. The finding that depleting RIF1, an antagonist of BRCA1, could not increase the number of BRCA1 IRIF suggests that either Pro-NHEJ sites contain an inhibitor(s) of BRCA1 recruitment or they lack polyubiquitination modification (mainly K63-linked). To test which possibility is more likely, we first co-stained $\gamma \mathrm{H} 2 \mathrm{AX}$ (to mark most if not all DSBs) and poly-ubiquitin with FK2 antibodies. As shown in Fig. 2a, while more than $90 \% \gamma \mathrm{H} 2 \mathrm{AX}$ foci were costained with FK2, not all $\gamma \mathrm{H} 2 \mathrm{AX}$ foci were positive for FK2 in S/ G2, only $70 \%( \pm 8.05 \%)$ on average (with a range of 47.5 to $87.5 \%)$. Further, while more than $90 \%( \pm 6.13 \%)$ of FK2 foci costained with RIF1 in G1 cells, the co-localization diminished in S/ G2 cells (Fig. 2b). Thus, the reason that Pro-NHEJ sites cannot recruit BRCA1, even in the absence of RIF1, is most likely the lack of poly-ubiquitin modification.

A necessary step in HDR is to resect the broken ends and generate ssDNA which is bound by RPA (replication protein $A)^{38}$. The bound RPA then initiates a series of events 
a

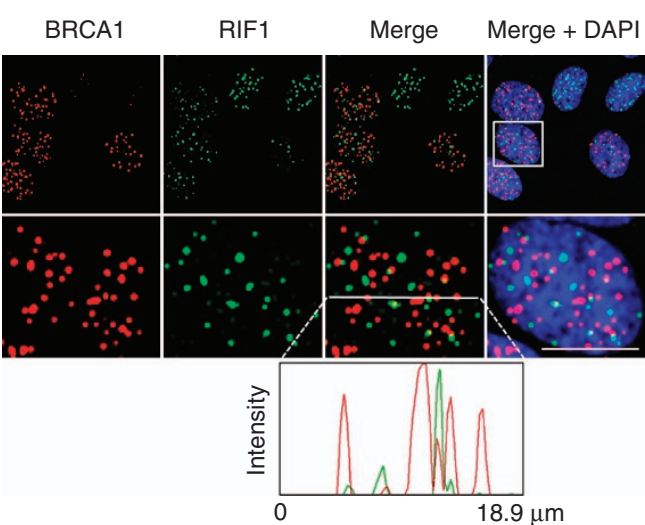

C
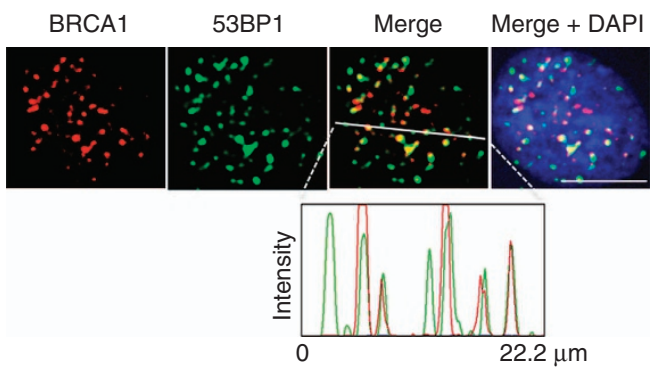

b
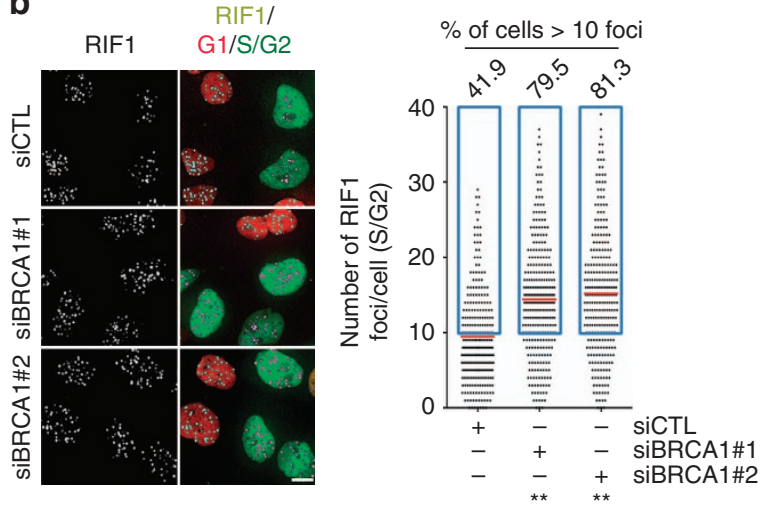

d
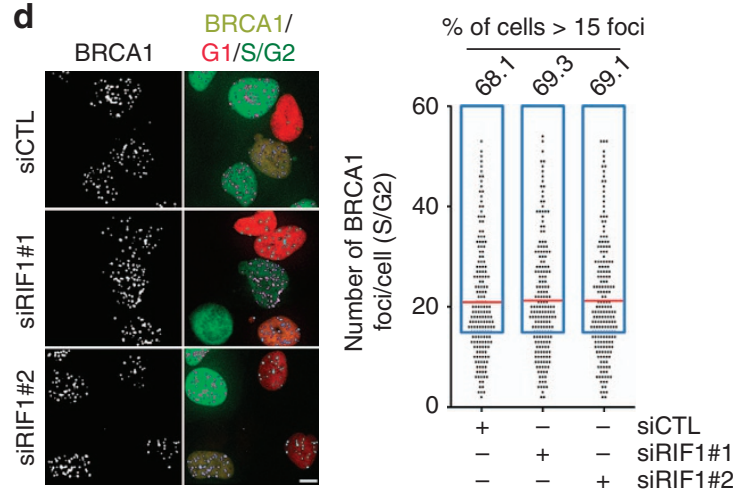

Figure 1 | RIF1 and BRCA1 IRIF co-exist in S/G2 cells. (a) Co-staining of BRCA1 and RIF1 in U2OS cells $1 \mathrm{~h}$ after 2 Gy IR. (b) Quantification of RIF1 focus number per cell in U2OS-Fucci cells transfected with control or two different BRCA1 siRNAs. The cells were subjected to 2 Gy IR 3 days after the siRNA transfection. Left panel: representative images of RIF1 foci. Right panel: quantification of RIF1 focus number in the cells $(n>212)$. (c) Co-staining of BRCA1 and 53BP1 in U2OS cells $1 \mathrm{~h}$ after 2 Gy IR. (d) Quantification of BRCA1 focus per cell in U2OS-Fucci cells transfected with control or two different RIF1 siRNAs. Cells were subjected to 2 Gy IR 3 days after the siRNA transfection. Left panel: representative images of BRCA1 foci. Right panel: quantification of BRCA1 focus number in the cells $(n>188)$. The intensity profile graphs show the immunofluorescence intensities of the indicated proteins along the line on the images $(\mathbf{a}, \mathbf{c})$. Red lines on the graphs indicate the mean number of RIF1 (b) or BRCA1 (d) foci per cell. Blue boxes designate cells with more than 10 RIF1 foci (b) or 15 BRCA1 foci (d). The percentages of RIF1-positive ( $>10$ foci) or BRCA1-positive ( $>15$ foci) cells are indicated above each blue box. ${ }^{\star \star}$ (in $\mathbf{b}$ ) indicates $P<0.001$ (student's $t$-test). Scale bars represent $10 \mu \mathrm{m}$.

including activation of ATR, Chk1, and the recruitment of BRCA1, BRCA2 and RAD51 (ref. 39). Therefore, RPA-decorated DSBs are intended for HDR or Pro-HDR, which implies colocalization of RPA IRIF with poly-ubiquitin chains. Indeed, costaining of RPA and poly-ubiquitin demonstrated overlapping between the two. As expected, RPA only partially overlapped with $\gamma \mathrm{H} 2 \mathrm{AX}$ foci (Fig. 2c), and RPA and RIF1 IRIF do not overlap (Fig. 2c).

These results indicate that DSBs in S/G2 cells are processed into two different kinds, one that possesses poly-Ub chains and is competent for BRCA1 recruitment (thus Pro-HDR), and another that does not and is incompetent for BRCA1 recruitment (but competent for RIF1, thus Pro-NHEJ). Based on the percentage of FK2-positive $\gamma \mathrm{H} 2 \mathrm{AX}$ foci (Fig. 2c), the ratio of Pro-HDR sites over Pro-NHEJ sites in a given cell was calculated to be 0.90 to 7.0 , with an average of 2.33 , indicating that HDR prevails in S/G2 cells. However, this range of ratios was obtained with U2OS cells. It may change in different types of cells.

USP1 prevents the accumulation of poly-Ub modification. The reason that the Pro-NHEJ DSB sites do not have poly-ubiquitination modification may be two-fold. One is that it lacks the E3 ligase RNF168 and another that the poly-Ub chains generated are actively destroyed in situ by a deubiquitinating enzyme (DUB).
We therefore looked for evidence that would support either of these two possibilities. Co-staining of $\gamma \mathrm{H} 2 \mathrm{AX}$ with RNF168 showed that the two overlapped more than $90 \%$ (Fig. 2d), suggesting that the first possibility is unlikely, which is consistent with the notion that RNF168 is also required for $53 \mathrm{BP} 1$ recruitment $^{25}$. For the second possibility, there are several DUBs including OTUB1, USP3, USP44 and POH1 that were reported to be able to remove K63-linked $\mathrm{Ub}$ at damage sites when ectopically expressed ${ }^{40-43}$, but apparently, none of them could do it at their endogenous levels, otherwise there would be no K63linked Ub present at DSBs at all. Thus, we thought that such a DUB must be recruited to DNA damage sites. To that end, we found USP1 was mobilized to DNA damage sites in an MDC1dependent, but RNF8-independent manner (Fig. 2e). Since the mobilization of MDC1 depends on ATM, the mobilization of USP1 also depends on ATM as expected (Supplementary Fig. 2a). However, USP3 and OTUB1, both reported to be able to inhibit $\mathrm{H} 2 \mathrm{~A}(\mathrm{X})$ ubiquitination ${ }^{40,41}$, did not show mobilization following microirradiation (Supplementary Fig. 2b). We further confirmed the recruitment of endogenous USP1 to microirradiated regions using USP1 specific antibodies (Supplementary Fig. 2c). We also found that the formation of K63-linked poly-ubiquitin chains at DNA damage sites was dramatically suppressed by ectopic expression of USP1 (Supplementary Fig. 2d) and such a suppression depended on its deubiquitinase activity as the 
expression of the catalytically inactive USP1 (C90S) had no effect (Supplementary Fig. 2e). In agreement with previous reports ${ }^{40,44}$, overexpression of OTUB1 and USP3 also highly inhibited K63 linkage formation following microirradiation (Supplementary Fig. 2f). In addition, we observed a marked reduction of H2AX poly-ubiquitination in cells overexpressing USP1 in an in vivo ubiquitination assay (Fig. 2f). USP1 at DSB sites very likely also removes K63-linked poly-Ubs formed on H1 (ref. 45) or K27linked Poly-Ubs ${ }^{45}$ as well to block BRCA1 recruitment. Indeed, ectopic expression of USP1 significantly inhibited BRCA1, but a

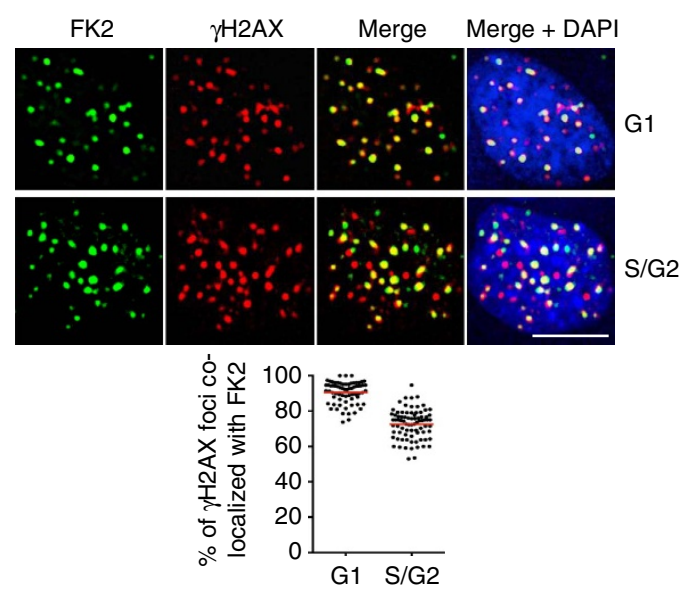

C

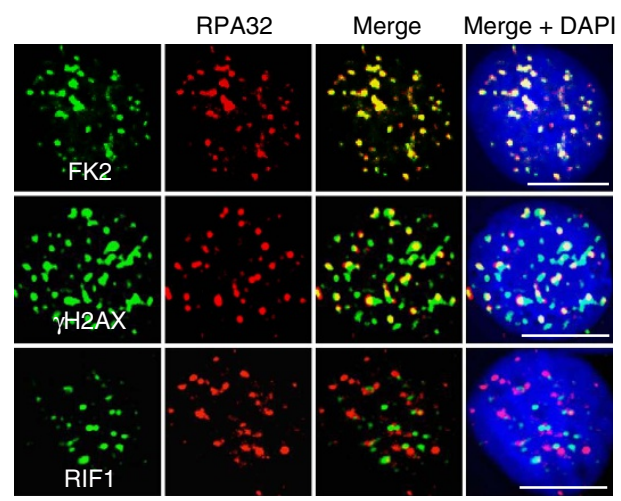

e

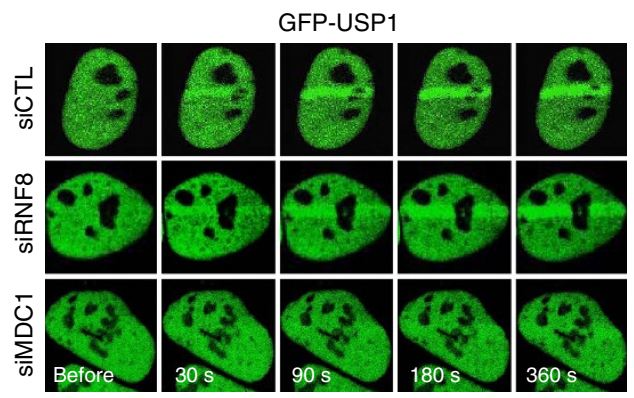

b

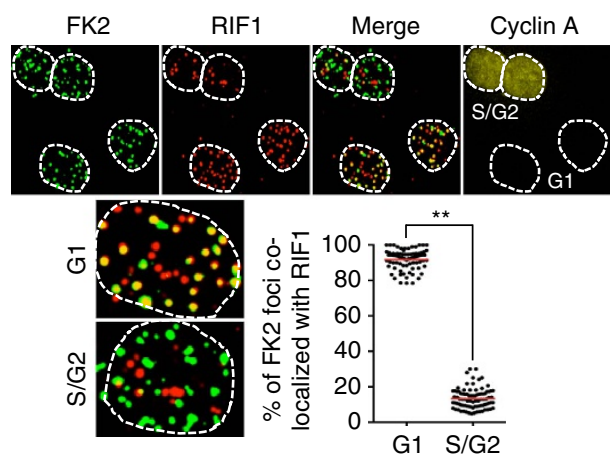

d

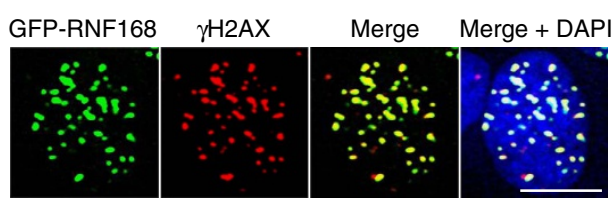

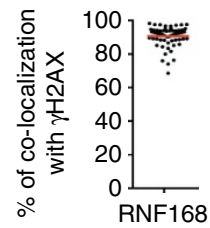

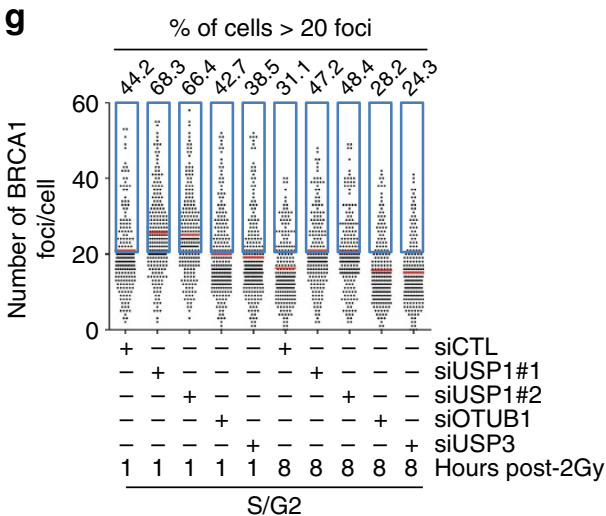

h

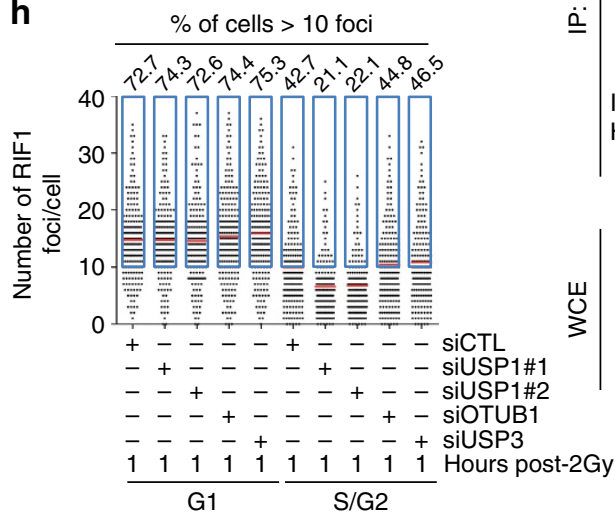

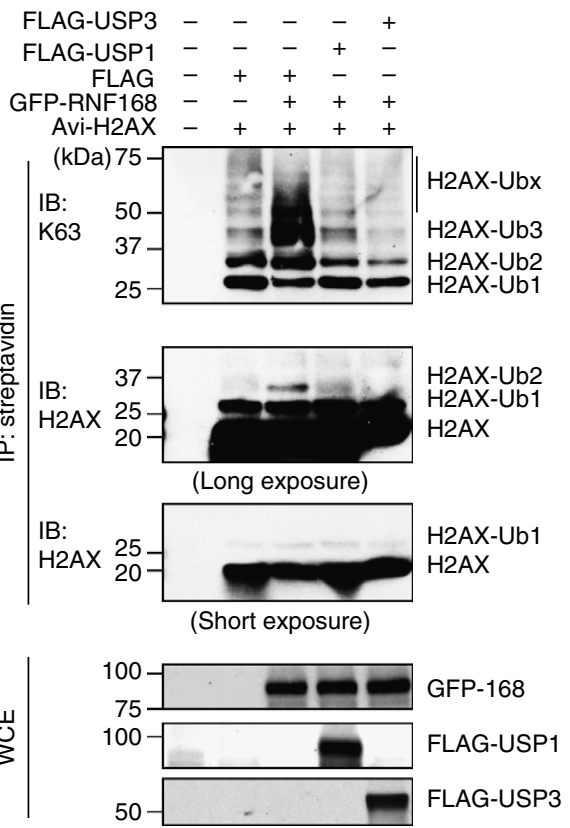


increased RIF1 focus formation in S/G2 cells (Supplementary Fig. 2g,h).

Unlike OTUB1 and USP3 which inhibited both BRCA1 and 53BP1 focus formation when overexpressed ${ }^{40,41}$, 53BP1 focus formation remained largely unaffected when USP1 was overexpressed (Supplementary Fig. 2i). In contrast to the overexpression, the depletion of USP1 increased the number of BRCA1 foci but decreased the number of RIF1 foci in S/G2 cells (Fig. 2g,h, Supplementary Fig. 2j,k). However, the depletion of either OTUB1 or USP3 had no significant effects on the focus formation of either BRCA1 or RIF1 in S/G2 cells (Fig. 2g,h, Supplementary Fig. 2j,k). These results suggest that USP1 is an inhibitor of DSB-induced chromatin ubiquitination in S/G2 cells. Given that the deubiquitinase activity of USP1 is highly regulated by its interacting partner, UAF1 (ref. 46), we found that the depletion of UAF1 with two distinct siRNAs also resulted in an increase of BRCA1 foci (Supplementary Fig. 2l), and at the same time a decrease of RIF1 foci in S/G2 cells (Supplementary Fig. $2 \mathrm{~m}$ ).

The anaphase-promoting complex functions at DNA damage sites. Since both Pro-HDR and Pro-NHEJ DSB sites co-exist in a single S/G2 cell, how then does the cell select which site to recruit USP1 (to erase poly-ubiquitination) and which site not to? Alternatively, a cell can make the selection by destroying USP1 at some sites but not at others. The latter seemed more attractive as we knew that USP1 was a substrate of $\mathrm{APC} / \mathrm{C}^{8}$. Specifically, USP1 is brought to APC for ubiquitination by one of its two adaptor proteins Cdh1 (Frr1). We reasoned that if USP1 was destroyed by APC ${ }^{\text {Cdhl } 1}$ at some DSB sites, this E3 complex itself must be able to go to DBS sites as well. Indeed, we found that APC and Cdh1 could be mobilized to DNA damage sites, and the mobilization was MDC1-dependent, but RNF8independent (Supplementary Fig. 3a,b). We also confirmed the recruitment of endogenous APC12 to microirradiated regions using APC12 specific antibodies (Supplementary Fig. 3c). Unfortunately, we were not successful to find Cdh1 antibodies suitable for immunofluorescent staining. RNF8 relocalization to sites of DNA damage was affected by neither Cdh1 nor APC3 depletion, indicating that RNF8 and APC ${ }^{\text {dh1 } 1}$ complex localize to DSBs independent of each other (Supplementary Fig. 3d). It was shown previously that Cdc27, a core subunit of APC, could be phosphorylated by ATM, which creates a phosphorylated motif at the C-terminus recognizable by $\mathrm{MDC1}$ (ref. 47). Thus, it is very likely that in response to DNA damage, Cdc27 is phosphorylated by ATM and the whole APC complex (with or without its adaptors) is then recruited to damage sites via the interaction between Cdc27 and MDC1. Consistent with that, Cdh1 depends on APC to be recruited, while APC recruitment does not require Cdh1 (Fig. 3a,b). Cdc20, another activator of APC, is required for neither APC nor Cdh1 recruitment to DSBs (Fig. 3a,b).

APC $^{\text {Cdh1 } 1}$ is active in G1 but is inactivated in S/G2 through interaction with the inhibitor Emil and the phosphorylation of
Cdh1 by cyclin-dependent kinases ${ }^{12,48}$. However, it has been previously reported that $\mathrm{APC}^{\mathrm{Cdh} 1}$ could be reactivated in response to DNA damage in S/G2 phase cells as evidenced by the increased binding of Cdh1 to APC ${ }^{10,49}$, the activity of purified APC/C from irradiated G2 cells in in vitro ubiquitination assays ${ }^{49}$, a shift of Cdh1 towards the less phosphorylated state following IR treatment in $2 \mathrm{D}$ gel analysis ${ }^{21}$, and a reduction of the expression of Emil induced by p53 and p21 (ref. 50). It is also possible that DNA damage may interrupt the interaction between Emil and APC Cdh1. To that end, we analysed the binding of Cdh1 to Emil. As expected, the binding between the two is significantly reduced following DNA damage in S/G2 phase cells (Fig. 3c).

It is known that APC catalyses the formation of K11-linked poly-Ub chains on its substrates ${ }^{6}$. To see if $\mathrm{APC}^{\mathrm{Cdh} 1}$ recruited to DNA damage sites is functional, we first determined whether APC-specific E2s were also recruited to damage sites. As shown in Supplementary Fig. 3e, both UBE2C and UBE2S could localize to microirradiated sites. Next, we stained for the presence of K11linked Ub. As shown in Fig. 3d,e, K11-linked Ub could be detected at damage sites either through staining of ectopically expressed tagged K11-only ubiquitin or through K11-linked Ubspecific antibodies. Interestingly, K11-linked Ub chains were accumulated to a much less extent at DNA damage sites in G1 than in S/G2 cells (Fig. 3d). Significantly, the K11-linked Ub signal diminished in APC3 as well as in Cdh1 depleted cells (Fig. 3e). These data suggest that APC ${ }^{\text {Cdh1 }}$ is a novel DNA damage response E3 ligase functioning in an RNF8-independent manner and promotes the formation of K11-linked Ub chains at DNA damage sites primarily in S/G2 cells.

USP1 is targeted by APC ${ }^{\text {Cdh1 } 1}$ at DSBs. If USP1 were the target of APC ${ }^{C d h 1}$ at damage sites, one would see the intensity of K11linked Ub signal diminishing in USP1-depleted cells. Indeed, as shown in Fig. 4a, the K11-linked Ub signal was close to undetectable in USP1 knockdown cells, suggesting that USP1 is the major, if not the only, substrate of APC ${ }^{2}$ hh1 at DNA damage sites. In agreement with that, the depletion of Cdh1 markedly prolonged the retention of USP1 at DNA damage sites (Fig. $4 \mathrm{~b}$ and Supplementary Fig. 4a).

A previous report has shown that USP1 is ubiquitinated by APC Cdh1 in an in vitro ubiquitination assay $^{8}$. Using K11-only mutant ubiquitin, we performed an in vivo ubiquitination assay of USP1 in S/G2 cells depleted of Cdh1. Figure 4c shows that USP1 was ubiquitinated (K11-linked) in vivo. Although the depletion of Cdh1 increased USP1 expression, the levels of ubiquitinated USP1 were significantly reduced, suggesting that the formation of K11 linkage on USP1 was highly dependent on the function of Cdh1.

Given that USP1 suppresses BRCA1, we expect that compromising APC function would also lead to an apparent suppression on BRCA1 focus formation. Indeed, the depletion of either APC3

Figure 2 | RIF1 IRIF in S/G2 cells lack poly-ubiquitination modification. (a) Co-staining of FK2 with $\gamma \mathrm{H} 2 \mathrm{AX}$ in U2OS cells $1 \mathrm{~h}$ after $2 \mathrm{~Gy}$ IR. The dot plot graph shows the percentages of $\gamma \mathrm{H} 2 \mathrm{AX}$ co-localization in the cells $(n>78)$. (b) Co-staining of FK2, RIF1 and cyclin A in U2OS cells $1 \mathrm{~h}$ after $2 \mathrm{~Gy}$ IR. Enlarged G1 and S/G2 images of FK2 and RIF1 co-staining are from the merged image in upper panel, and the graph shows the percentages of FK2 foci positive for RIF1 in G1 and S/G2 cells $(n>87)$. (c) Co-staining of FK2, $\gamma \mathrm{H} 2 \mathrm{AX}$ or RIF1 with RPA32 in U2OS cells. After $2 \mathrm{~Gy}$ IR, the cells were pre-extracted before immunofluorescence staining of the indicated proteins. (d) Co-localization of GFP-tagged RNF168 with $\gamma \mathrm{H} 2 \mathrm{AX}$. U2OS cells transfected with GFPtagged RNF168 expression plasmid were irradiated with $2 \mathrm{~Gy}$ and harvested $1 \mathrm{~h}$ later for analysis. The dot plot graph shows the percentages of $\gamma \mathrm{H} 2 \mathrm{AX}$ colocalization with RNF168 in the cells $(n>78)$. (e) Mobilization of USP1 to sites of DNA damage. U2OS cells transfected with GFP-USP1 and the indicated siRNAs were microirradiated with laser, and imaged. (f) In vivo ubiquitination of H2AX. HeLa cells (stably expressing NLS-BirA and Avi-tagged H2AX or NLS-BirA alone) were co-transfected with GFP-RNF168 and FLAG-USP1 or FLAG-USP3, irradiated and analysed. (g,h) Quantification of BRCA1 ( $>170$ cells analysed) (g) and RIF1 (>190 cells analysed) (h) focus number in U2OS-Fucci cells transfected with the indicated siRNAs. Blue box designates the cells with more than 20 (BRCA1 positive) and 10 (RIF1 positive) foci, respectively. The percentages of BRCA1-positive and RIF1-positive cells are indicated above each box. ${ }^{\star \star}$ (in $\mathbf{b}$ ) indicates $P<0.001$ (student's $t$-test). Scale bars represent $10 \mu \mathrm{m}$. 
a
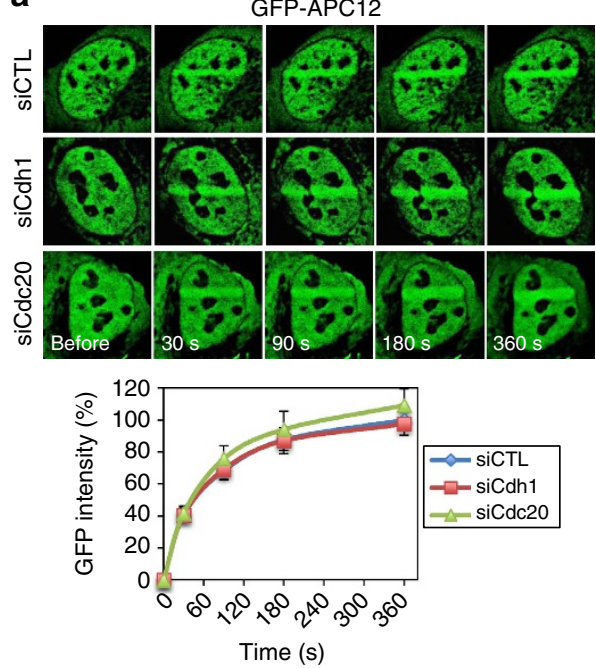

C

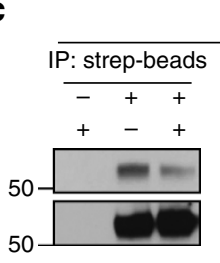

S/G2

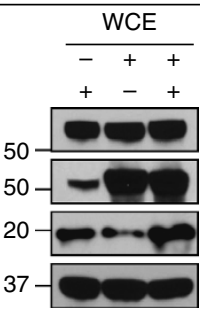

Strep-Cdh1 1h post 10 Gy b
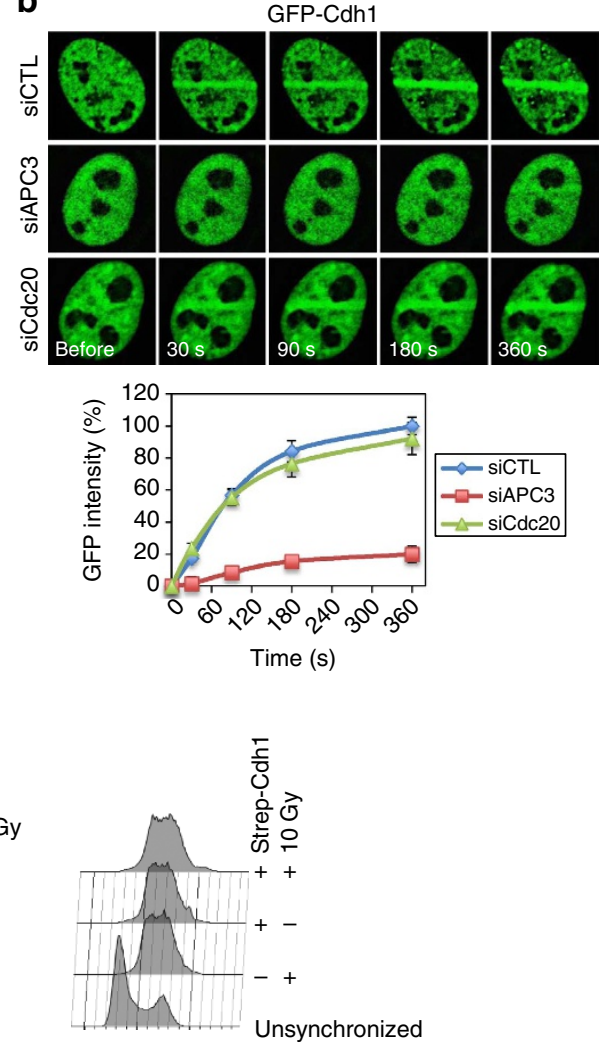

d

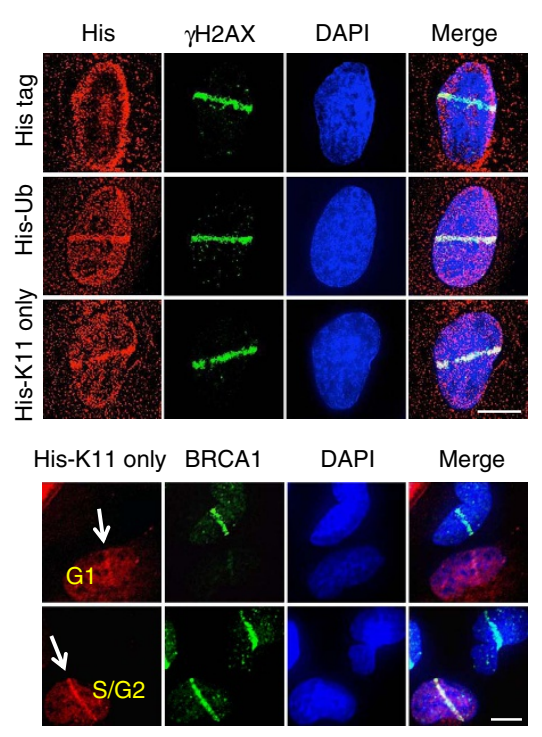

e
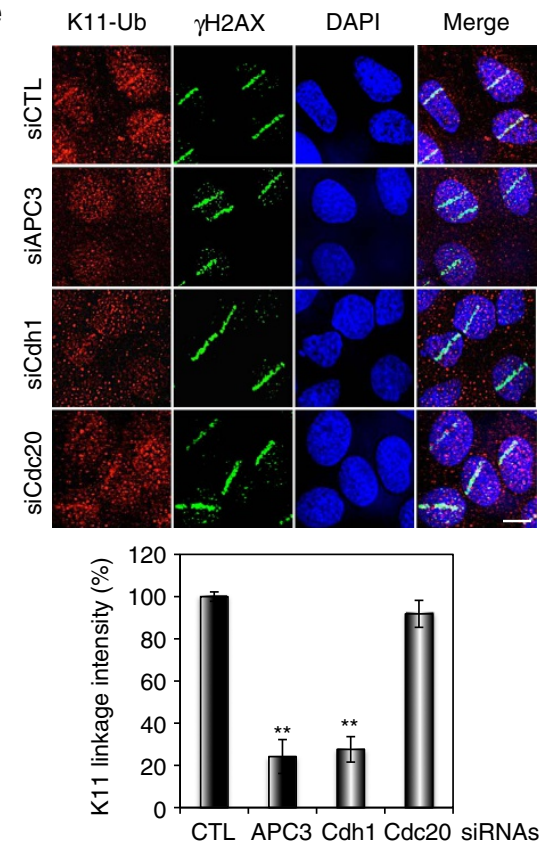

Figure 3 | The anaphase promoting complex protects poly-ubiquitin signalling at DSBs. (a,b) Mobilization of APC12 (a) and Cdh1 (b) to sites of DNA damage. U2OS cells were transfected with GFP-APC12 or GFP-Cdh1 expression plasmids and the indicated siRNAs, microirradiated with laser and imaged at different time points. (c) Interaction of Cdh1 with Emi1 in S/G2 cells. HeLa cells stably expressing strep-tagged Cdh1 were synchronized with double thymidine, released into $S / G 2$, exposed to $10 \mathrm{~Gy} I R$ and collected after $1 \mathrm{~h}$ recovery for immunoprecipitation analysis. (d) The accumulation of K11-linked poly-ubiquitin at sites of DNA damage. U2OS cells were transfected with ubiquitin (Ub) constructs, microirradiated and processed 30 min later for immunostaining of His tag and BRCA1. (e) The accumulation of the endogenous K11-linked Ub chains at sites of DNA damage. Immunostaining of K11linked $\mathrm{Ub}$ and $\gamma \mathrm{H} 2 \mathrm{AX}$ was performed $30 \mathrm{~min}$ after microirradiation. Top: representative images of K11-linked poly-(Ub) and $\gamma \mathrm{H} 2 \mathrm{AX}$ immunostaining in U2OS cells transfected with the indicated siRNAs. Bottom: quantification of K11 linkage intensity at microirradiated regions ( $>110$ cells quantificated). Error bars indicate SEM from three independent experiments. ${ }^{\star *}$ (in $\mathbf{e}$ ) indicates $P<0.001$ (student's $t$-test). Scale bars represent $10 \mu \mathrm{m}$. 
a

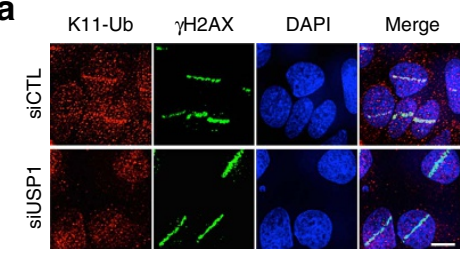

C

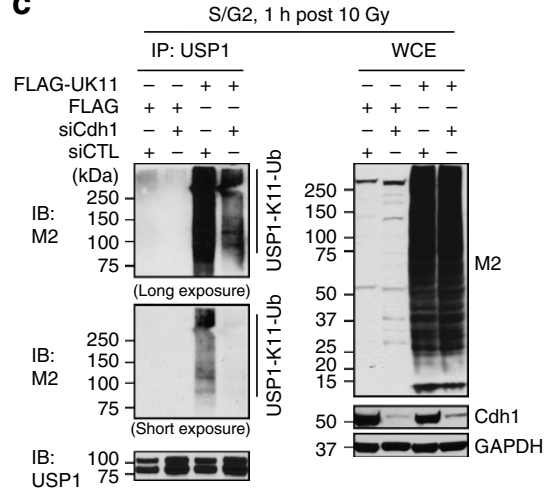

b

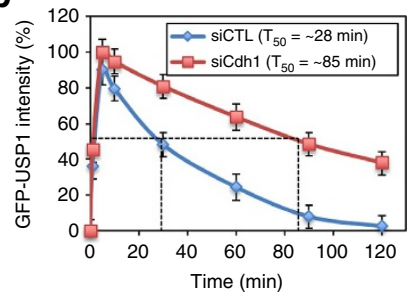

d
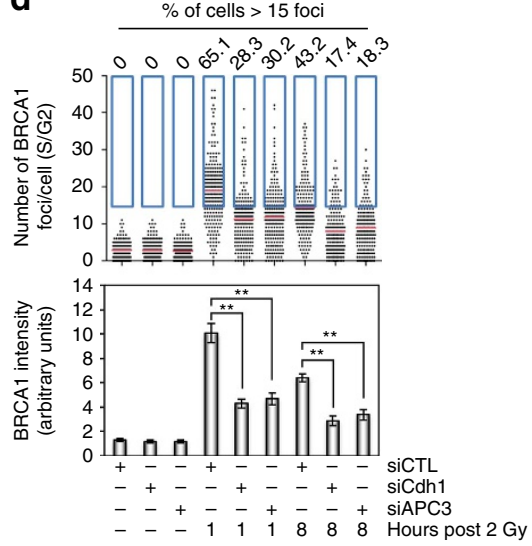

h

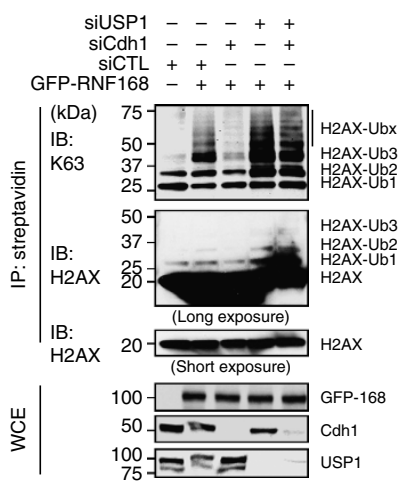

$\mathbf{f}$

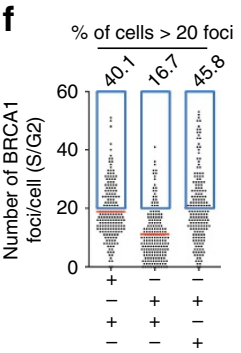

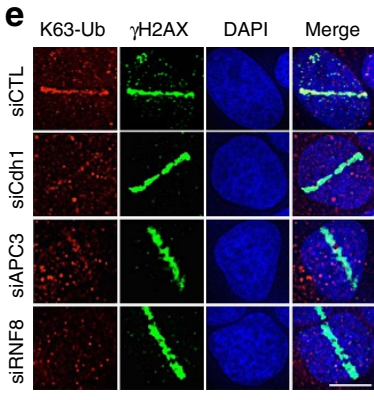

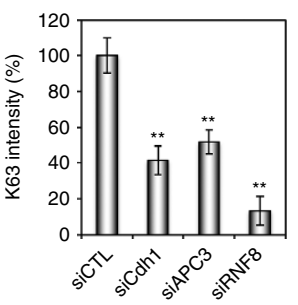

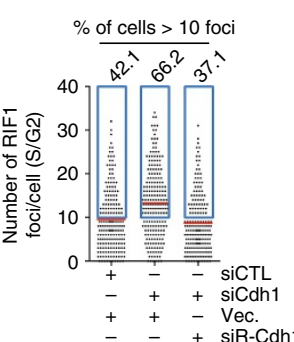

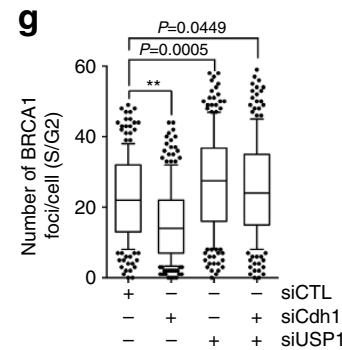

Figure 4 | USP1 is targeted by APC ${ }^{\text {Cdh1 } 1}$ at DSBs. (a) The K11-linked ubiquitin chains were primarily formed on USP1. U2OS cells transfected with control or USP1 siRNA were microirradiated and processed for immunostaining 30 min later. (b) The mobilization kinetics of GFP-USP1 to sites of DNA damage. GFPUSP1 was expressed in U2OS cells transfected with the indicated siRNAs and its mobilization was monitored for $2 \mathrm{~h}$ following the microirradiation. (c) In vivo ubiquitination assay of endogenous USP1 in the presence or absence of Cdh1 after DNA damage. Cell lysates were prepared as described in methods. (d) Quantification of BRCA1 focus number and intensity in Cdh1- and APC3-depleted cells. The cells were subjected to 2 Gy IR 3 days after the siRNA transfection. Top panel: quantification of BRCA1 foci per cell. Blue box designates the cells with more than 15 foci, whose percentage is indicated above each box. Bottom panel: average intensities of BRCA1 focus per cell $(n>195)$. (e) The accumulation of endogenous K63-linked ubiquitin chains at damage sites. U2OS cells were microirradiated and recovered for $30 \mathrm{~min}$ before the immunofluorescence analysis of K63-linked Ub and $\gamma \mathrm{H} 2 \mathrm{AX}$. Left panel: representative images of K63-linked Ub and $\gamma \mathrm{H} 2 \mathrm{AX}$. Right panel: quantification of K63-linked Ub at microirradiated regions $(n>102)$. (f) Quantification of BRCA1 ( $n>191)$ and RIF1 ( $n>197$ ) focus formation in U2OS-Fucci cells transfected with the indicated siRNAs and siCdh1-resistant Cdh1 constructs. Blue box designates the cells with more than 20 (BRCA1 positive) and 10 (RIF1 positive) foci, respectively. The percentages of BRCA1-positive and RIF1-positive cells are indicated above each box. (g) Quantification of BRCA1 focus number in the cells transfected with the indicated siRNAs $(n>200)$. (h) In vivo ubiquitination of H2AX in Cdh1-, USP1- or both Cdh1- and USP1-depleted cells. The indicated siRNAs were transfected first, followed by GFP-RNF168 transfection $48 \mathrm{~h}$ later, and another $24 \mathrm{~h}$ later, the cells were irradiated with $10 \mathrm{~Gy}$ and harvested $1 \mathrm{~h}$ after IR for the analysis of H2AX ubiquitination. Error bars indicate SEM from three independent experiments. ${ }^{\star \star}$ indicates $P<0.001$ (student's $t$-test). Scale bars represent $10 \mu \mathrm{m}$. 
or Cdh1 caused a severe reduction in the number and the intensity of BRCA1 foci as well as a similar decrease in K63linked Ub signal strength at micro-irradiated regions (Fig. 4d,e, and Supplementary Fig. 4b). The depletion of Cdh1 via shRNA targeting a different region of Cdh1 sequence also resulted in a reduction of BRCA1 and Rad51 foci as well as a reduction of the K63-linked poly-(Ub) at DNA damage sites (Supplementary Fig. 4c). Furthermore, both BRCA1 and RIF1 focus formation were restored to normal by the ectopic expression of siRNAresistant Cdh1 in the cells depleted of the adaptor (Fig. $4 \mathrm{f}$ and Supplementary Fig. 4d,e,f), indicating that the effects of Cdh1 depletion on BRCA1 and RIF1 focus formation we observed were not mediated by off-target effects of the siRNA. More importantly, the simultaneous depletion of Cdh1 and USP1 restored near completely the focus formation of BRCA1 and Rad51, as well as the accumulation of K63-linked Ub chains (Fig. $4 \mathrm{~g}$ and Supplementary Fig. 4g,h,i). Consistent with these immunostaining results, we observed a significant decrease of H2AX ubiquitination in Cdh1-depleted cells, but an increase in USP1-depleted cells (Fig. 4h).

To further establish the role of APC ${ }^{\mathrm{Cdh} 1}$ in antagonizing USP1 at DNA damage sites, we generated U2OS cell lines that inducibly express USP1, catalytically inactive USP1 (C90S mutant), and APC $^{\text {Cdh1 }}$-resistant USP1 ( $\triangle 295-342$ mutant $)^{8}$ at the endogenous level of USP1 expression plus a line with the expression vector only as a control (Supplementary Fig. 5). All three forms of USP1 expressed were made siUSP1 resistant. These cells were then depleted of USP1 and examined for their ability to form BRCA1 and RIF1 IRIF. As shown in Fig. 5, the depletion of USP1 in control cells (no ectopic USP1 expression) expectedly caused an increase of BRCA1 IRIF but a decrease of RIF1 IRIF (as seen in Fig. 2g,h). These effects were largely eliminated by re-expressing wild-type USP1, but not by re-expressing the catalytically inactive USP1. In sharp contrast, however, USP1 ( $\Delta 295-342)$-expressing cells showed greatly diminished ability to form BRCA1 foci but at the same time showed greatly enhanced ability to form RIF1 foci (Fig. 5a-d), as if these cells were overexpressing wild-type USP1 (see Supplementary Fig. 2g,h). Thus, expression of APC Cdh1 resistant USP1 at endogenous levels favours the generation of Pro-NHEJ sites at the expense of Pro-HDR sites.

Chk1-Cdc14B signalling is required for Cdh1 recruitment. Both APC complex and USP1 depend on MDC1 to be recruited to DNA damage sites (Supplementary Fig. 3a, Fig. 2e), but only the Pro-HDR sites seem to be able to activate APC and destroy USP1 (Fig. 4g). One must wonder what is present at Pro-HDR sites but absent at Pro-NHEJ sites that could activate APC, therefore protecting the poly-ubiquitin signal required for BRCA1 recruitment. We reasoned that by examining the requirement of a

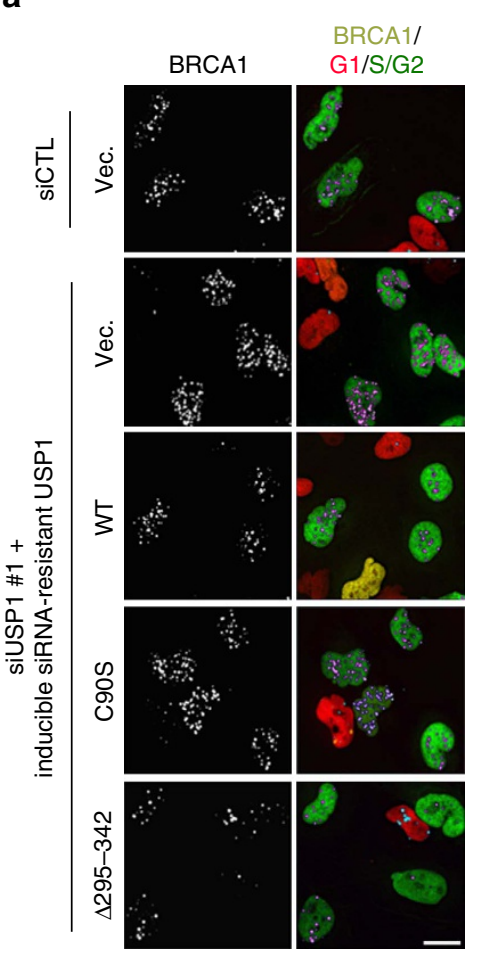

b

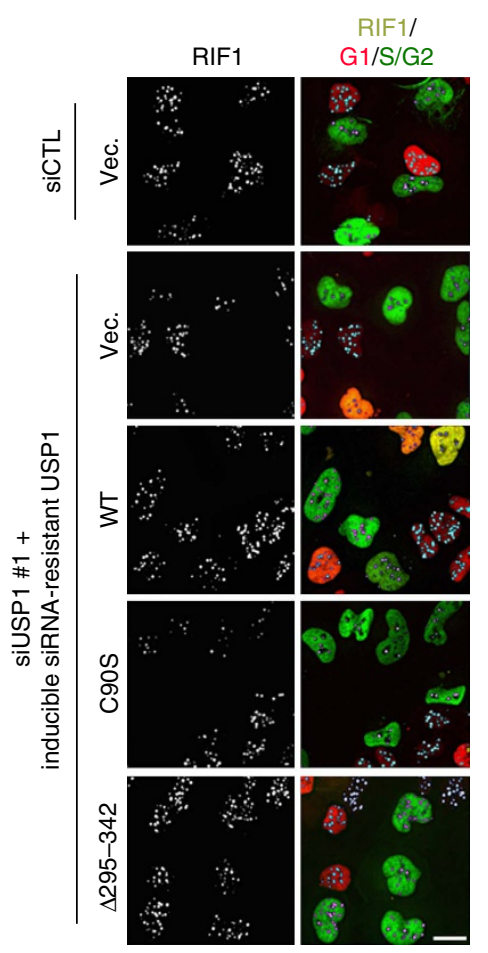

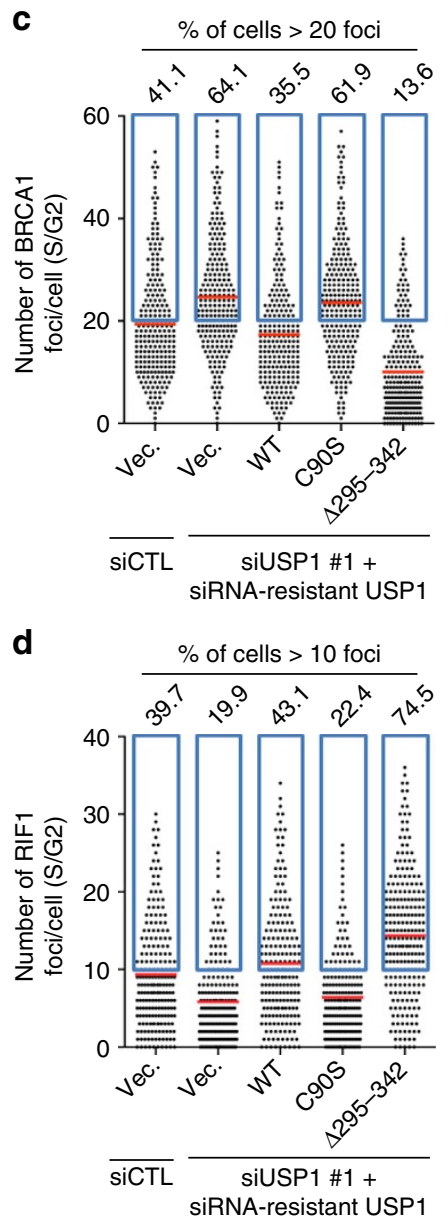

Figure 5 | Antagonizing USP1 by APC ${ }^{\text {Cdh1 }}$ at DNA damage sites is critical for BRCA1 recruitment. (a,b). Representative images of BRCA1 (a) and RIF1 (b) IRIF in U2OS-Fucci cells transfected with the indicated siRNAs and expression vectors. The cells were irradiated with $2 \mathrm{~Gy}$ and recovered for $1 \mathrm{~h}$ before the immunostaining. (c,d) Quantification of BRCA1 (c, n>226) and RIF1 (d, $n>195)$ focus formation in a,b. Blue box designates cells with more than 20 (BRCA1 positive) and 10 (RIF1 positive) foci, respectively. The percentages of BRCA1-positive and RIF1-positive cells are indicated above each box. Scale bars represent $10 \mu \mathrm{m}$. 
known DDR signalling regulators in the recruitment of the APC activator Cdh1, we might unravel clues to that question. To that end, we found that Cdh1 recruitment not only depended on ATM similarly as APC complex itself (Fig. 6a), but it also required Chk1 (Fig. 6a,b). However, neither APC (APC12) (Fig. 6c) nor
USP1 (Fig. 6d) required Chk1. In these experiments, ATM and Chk1 kinases was inactivated via either siRNA or chemical inhibition.

We and others have shown previously that $\mathrm{Cdc} 14$ lies upstream of Cdh1 in DNA damage repair, likely through a
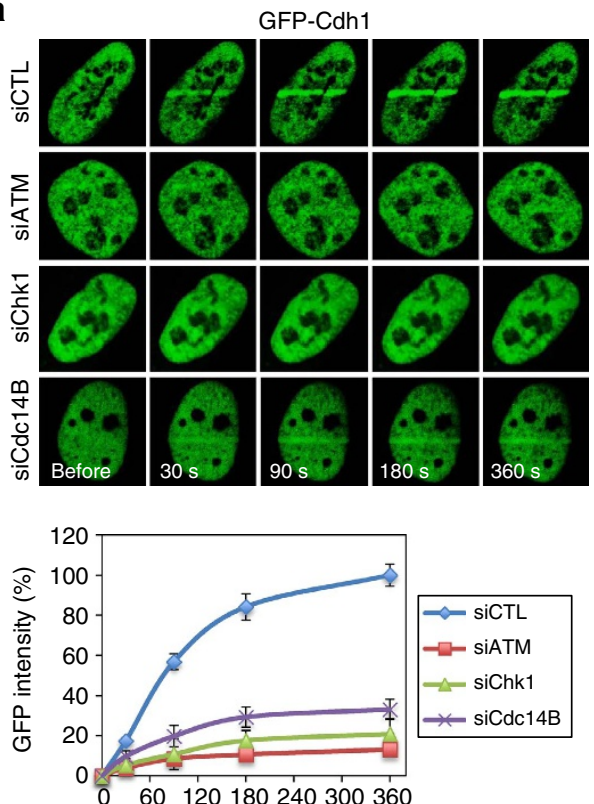

e

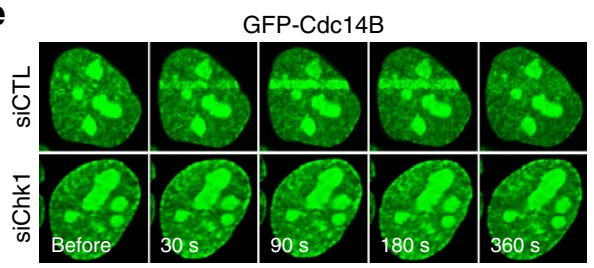

$\mathbf{f}$
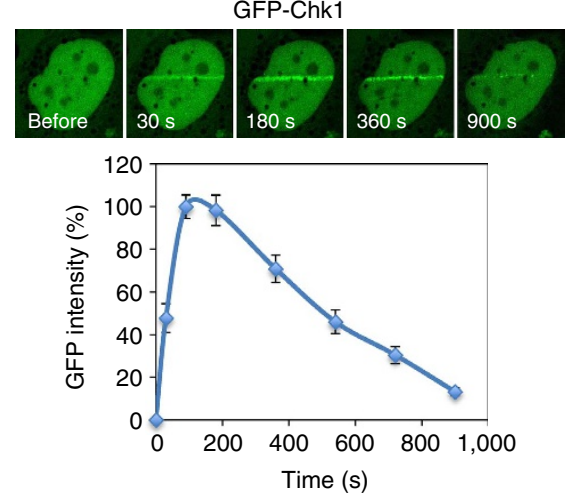

b

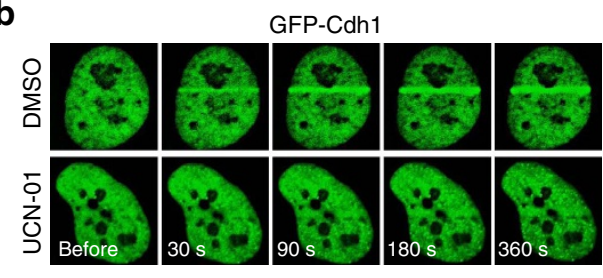

C

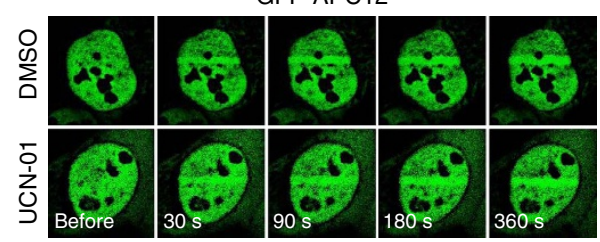

d

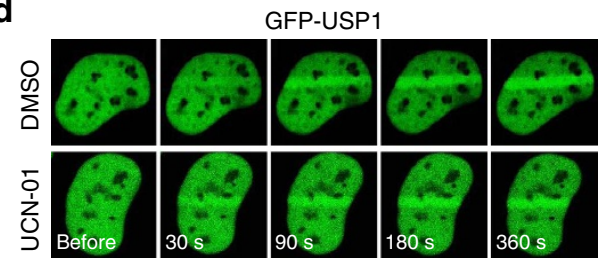

g
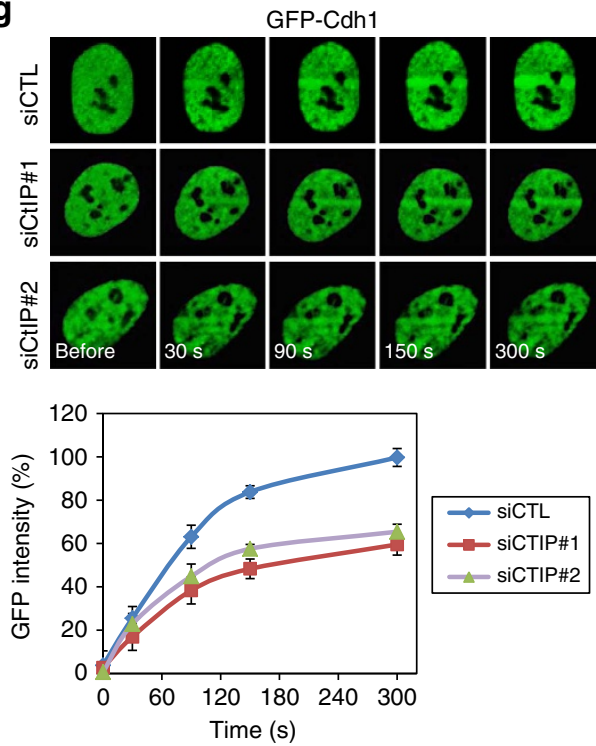

h

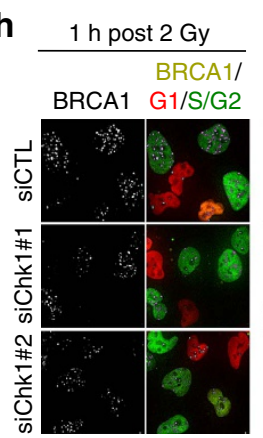

$\frac{8 \mathrm{~h} \text { post } 2 \mathrm{~Gy}}{\text { BRCA1/ }}$

BRCA1 G1/S/G2

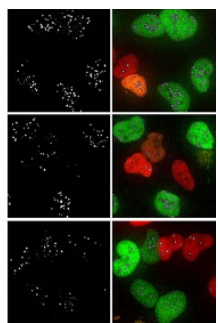

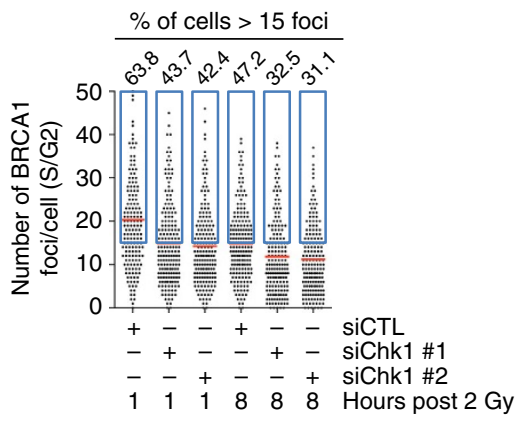


dephosphorylating (hence activating) Cdh1 (refs 10,21), and consistent with that, we found that the recruitment of Cdh1 to DNA damage sites largely depended on Cdc14B (Fig. 6a), whereas APC did not (Supplementary Fig. 6a). It has been

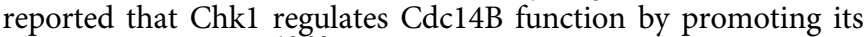
release from nucleoli ${ }^{10,22}$. Thus, it is likely that Chk1 regulates the localization of $\mathrm{Cdh} 1$ by releasing $\mathrm{Cdc} 14 \mathrm{~B}$ from nucleolus to nucleoplasm where the phosphatase can then act on Cdh1. Interestingly, Cdc14B is also localized to damage sites (although transiently, for about $5 \mathrm{~min}$ ) and the localization depends on Chk1 (Fig. 6e), suggesting that Cdh1 dephosphorylation and activation may be limited to DNA damage sites where Cdc14B is present. Given that GFP-Cdc14B was already present in the nucleoplasm in our experiment due to overexpression (Fig. 6e), the function of Chk1 in the localization of Cdc14B to damage sites is unlikely just to release the phosphatase from nucleoli. It probably also actively helps the mobilization of the phosphatase to damage sites. Consistent with that, we have shown previously that Cdc14B interacts with Chk1 (ref. 22). Moreover, Chk1 itself was localized to damage sites (Fig. 6f). In agreement with the finding that Chk1 is released from damage sites soon after activation $^{51,52}$, we found Chk1's presence at damage sites peeked by 2 min which strikingly coincide with the duration of Cdc14B at damage sites (Fig. 6e), reinforcing the conclusion that Chk1 brings the phosphatase to damages sites.

Given that Chk1 is activated by ssDNA-RPA-ATR signalling in which ssDNA is the product of end resection in preparation of the DSB for $\mathrm{HDR}^{39}$, we asked if CtIP, a key end resection factor along with MRN complex ${ }^{53}$, is required for Cdh1 recruitment. Indeed, as shown in Fig. 6g, depletion of CtIP substantially reduced the amount of Cdh1 recruited to DNA damage sites. As a result, the accumulation of poly-Ubs was also reduced significantly (Supplementary Fig. 6b).

Next, we determined whether Chk1 regulated accumulation of BRCA1 at sites of DNA damage. Chk1 was depleted in U2OSFucci cells with two distinct siRNAs and the cells were subjected to $2 \mathrm{~Gy} \gamma$-irradiation. As expected, BRCA1 IRIF was significantly inhibited in Chk1-depleted cells (Fig. 6h). Taken together, these results strongly suggest that there is a separate and Chk1dependent pathway, in addition to the one that recruits APC, that regulates Cdh1 localization and activation at Pro-HDR sites. Since Chk1 is activated by ssDNA-RPA-ATR signalling in which ssDNA is the product of end resection in preparation of the DSB for $\mathrm{HDR}^{39}$, the requirement of Chk1 links Cdh1 recruitment to end resection, and provides additional support to the notion that Chk1 is required for homologous recombination in mammalian cells ${ }^{54}$.

Cdh1 is required for HDR repair of DSBs. Having established that the recruitment of BRCA1 requires the function of APC ${ }^{\mathrm{Cdh} 1}$, we expected that the deficiency in Cdh1 would compromise HDR. We therefore examined the HDR efficiency in U2OS cells carrying a HDR reporter ${ }^{21}$. The depletion of Cdh1 caused a reduction in HDR close to that caused by BRCA1 depletion, but the depletion of USP1, as expected, resulted in an apparent increase in the HDR efficiency (Fig. 7a). More importantly, the depletion of USP1 restored the reduction in HDR efficiency caused by compromised Cdh1 function, reinforcing the notion that APC Cdh1 antagonizes USP1. Under these conditions, we did not observe significant effects exerted by Cdh1, USP1 or BRCA1 depletion (Supplementary Fig. 7a), indicating that the differences in HDR efficiencies are unlikely caused by disturbance in cell cycle phase distribution. Further, re-expression of siRNAresistant and APC $^{\text {Cdh1 } 1}$-resistant USP1 in USP1-depleted U2OS cells severely blocked HDR repair (Supplementary Fig. 7b), mimicking the loss of Cdh1 (Fig. 7a). In contrast, the depletion of USP1 resulted in a decrease in the NHEJ efficiency (Fig. 7b), and the re-expression of $\mathrm{APC}^{\mathrm{Cdh} 1}$-resistant USP1 increased the efficiency as expected (Supplementary Fig. 7c). Interestingly, Cdh1 depletion reduced the NHEJ efficiency as well, suggesting that Cdh1 might also regulate factors involved in NHEJ repair in G1. Moreover, mouse embryonic fibroblasts (MEFs) with Cdh1 deleted showed a delay in repairing DSBs (Fig. 7c) and defects in forming Rad51 IRIF (Fig. 7d). Taken together, these results demonstrate the importance of the interplay between $\mathrm{APC}^{\mathrm{Cdh} 1}$ and its substrate USP2 in the repair of DSBs.

\section{$\mathrm{APC}^{\mathrm{Cdh} 1}$ antagonizes USP1 in response to replication stress.} Replication stress presents a unique problem to cells as it often results in replication fork collapse which in turn generates DSBs. These DSBs are often one-ended and can only be repaired through $\mathrm{HDR}^{55,56}$. To determine if Cdh1 plays a similar role in dealing with replication stress as with ionizing radiation, we first determined the sensitivity of the cells depleted of Cdh1 to hydroxyurea (HU) treatment. As shown in Fig. 7e, Cdh1-depleted cells displayed similar sensitivity as BRCA1-depleted cells, indicating that $\mathrm{APC}^{\mathrm{Cdh} 1}$ is required for HDR of DSBs resulted from collapsed replication forks. As expected, the number of BRCA1 foci reduced dramatically in Cdh1-depleted cells treated with HU, and the simultaneous depletion of USP1 with that of Cdh1 essentially restored BRCA1 focus number (Fig. 7f). Interestingly, in contrast to IR-treatment, the depletion of USP1 alone in HU-treated cells had no effect on BRCA1 foci (Fig. 7f) nor on HU sensitivity (Fig. 7e), which is consistent with the notion that DSBs resulted from replication stress are mostly dealt with HDR, not NHEJ (thus are exclusively Pro-HDR) ${ }^{55,56}$. Indeed, unlike BRCA1, RIF1 focus formation was not induced by $\mathrm{HU}$ treatment (Fig. $7 \mathrm{~g}$ ). However, the depletion of Cdh1 did cause an increase in RIF1 focus number in HU-treated cells, and the double depletion of Cdh1 and UPS1 turned that number back to 'normal' (Fig. 7g), further supporting the conclusion that there is a competition between BRCA1 and RIF1 at DSBs and the competition is swung one way or another by USP1 and APC Cdh1 in S/G2 cells. Moreover, compromising Chk1 function via siRNA

Figure 6 | Chk1-Cdc14B signalling is required for Cdh1 recruitment. (a) The mobilization of Cdh1 to sites of DNA damage. U2OS were transfected with GFP-Cdh1 and the indicated siRNAs, laser microirradiated and imaged. The graph shows the intensities at the irradiated regions of GFP-Cdh1 over time. (b) The effect of Chk1 inhibitor UCN-01 on mobilization of Cdh1 to sites of DNA damage. Twenty-four hours after the transfection of GFP-Cdh1, the cells were treated with UCN-01 $(300 \mathrm{nM})$ for another $24 \mathrm{~h}$ followed by microirradiation. (c,d) The effect of UCN-01 on mobilization of APC12 (c) and USP1 (d) to sites of DNA damage. The cells were similarly treated as those in $\mathbf{b}$. (e) The mobilization of Cdc14B to sites of DNA damage. The experiment was performed similarly as in a. (f) The mobilization of Chk1 to sites of DNA damage. Top panel: representative images of GFP-Chk1 recruitment following microirradiation; bottom panel: quantification of GFP-Chk1 intensity at the damaged regions. The cells used were U2OS-Fucci or U2OS synchronized to S/ G2 through double thymidine block and release. (g) The mobilization of Cdh1 to microirradiated regions is suppressed by CtIP depletion. (h) Quantification of IR-induced BRCA1 focus formation in U2OS-Fucci cells transfected with control and two different Chk1 siRNAs. Three days after the siRNA transfection, immunostaining was performed at 1 and $8 \mathrm{~h}$ post $2 \mathrm{~Gy}$ of IR. Left panel: representative images of BRCA1 foci. Right panel: quantification of BRCA1 focus number in the cells $(n>174)$. Error bars indicate s.e.m. 


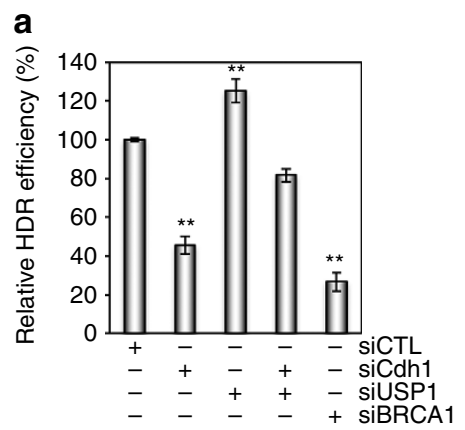

d
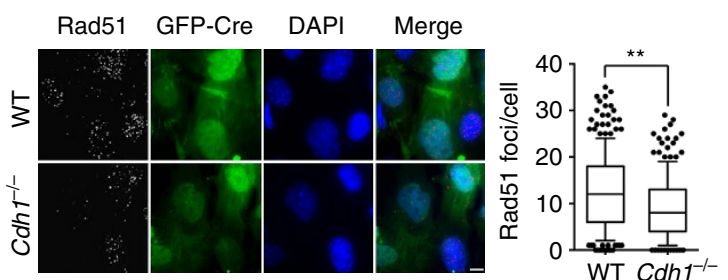

b

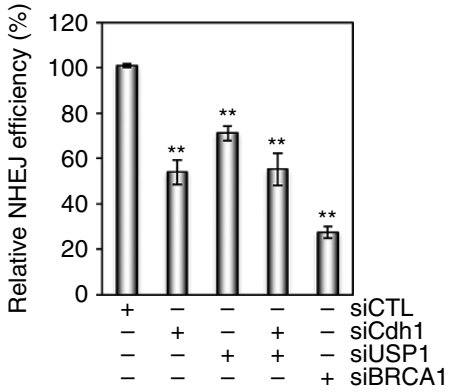

c

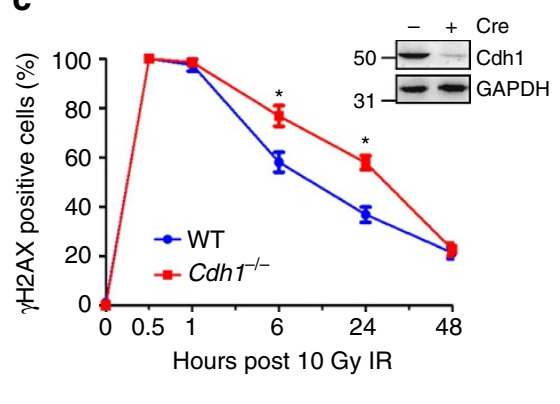

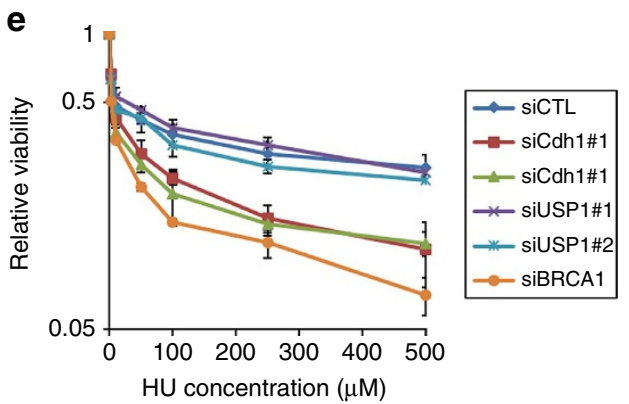

$\mathbf{f}$
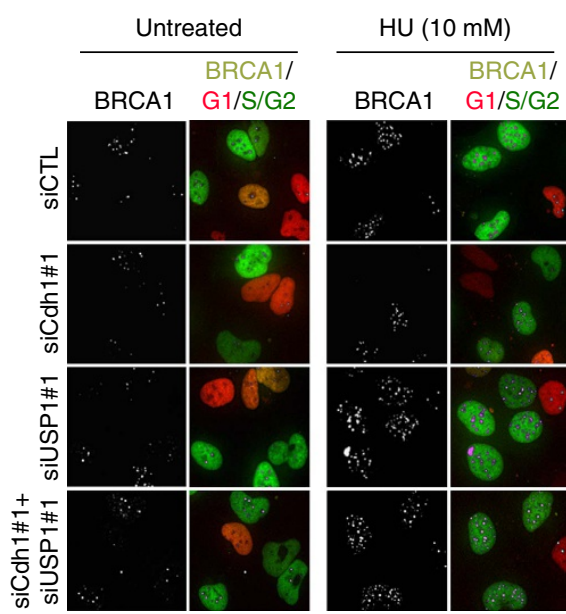

g
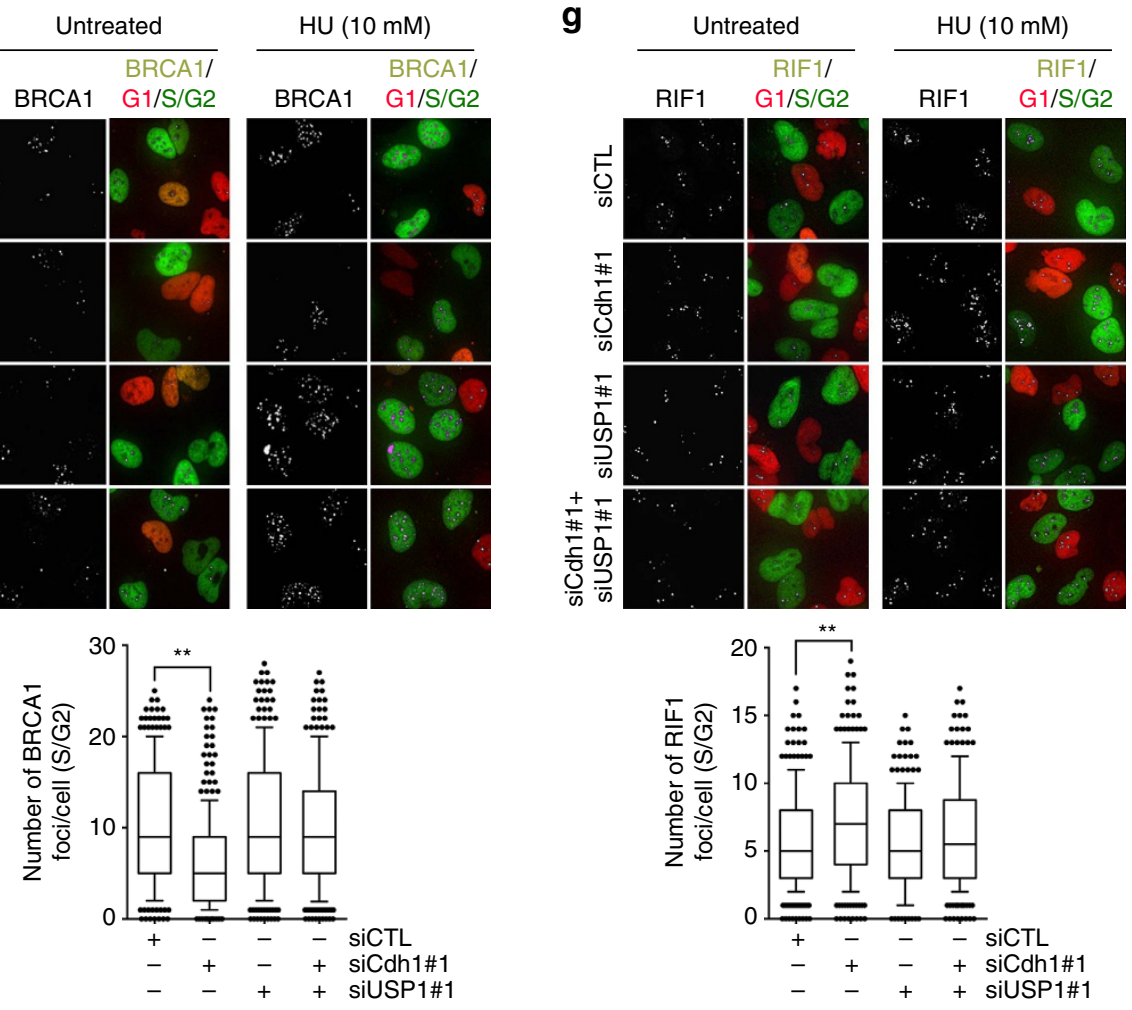

Figure 7 | Cdh1 is required for HDR repair of DSBs. (a,b) Quantification of HDR (a) and NHEJ (b) assays in cells transfected with indicated siRNAs. The HDR (the percentage of GFP + cells among mCherry + cells) and NHEJ (the percentage of tdTomato + cells among GFP + cells) efficiencies in cells transfected with the indicated siRNAs were normalized to that in control (siCTL) cells. (c) Quantification of $\gamma \mathrm{H} 2 \mathrm{AX}$ foci in WT and Cdh1 ${ }^{-/-}$MEFs after 10 Gy IR. (d) Immunostaining of Rad51 in WT and Cdh $7^{-/}$- MEFs after 10 Gy IR. The cells were fixed $1 \mathrm{~h}$ after the irradiation, and processed for immunofluorescence analysis. (e) Survival of U2OS cells transfected with the indicated siRNAs after hydroxyurea treatment. Twenty four hours after transfection of the siRNAs, the cells were treated with the indicated concentrations of $\mathrm{HU}$ for $24 \mathrm{~h}$ and then cultured in fresh media for additional 2 days before the viability test. $(\mathbf{f}, \mathbf{g})$ Immunofluorescence analysis of BRCA1 (f) and RIF1 ( $(\mathbf{g})$ in U2OS-Fucci cells transfected with the indicated siRNAs. Three days after the siRNA transfection, the cells were treated with $10 \mathrm{mM} \mathrm{HU}$ for $2 \mathrm{~h}$ and collected for the analysis. Top panel: representative images of BRCA1 and RIF1 foci in cells transfected with indicated siRNAs. Bottom panel: quantification of BRCA1 and RIF1 focus number per cell $(n>218)$. Error bars indicate SEM. ${ }^{\star \star}$ indicates $P<0.001$ (student's $t$-test). 


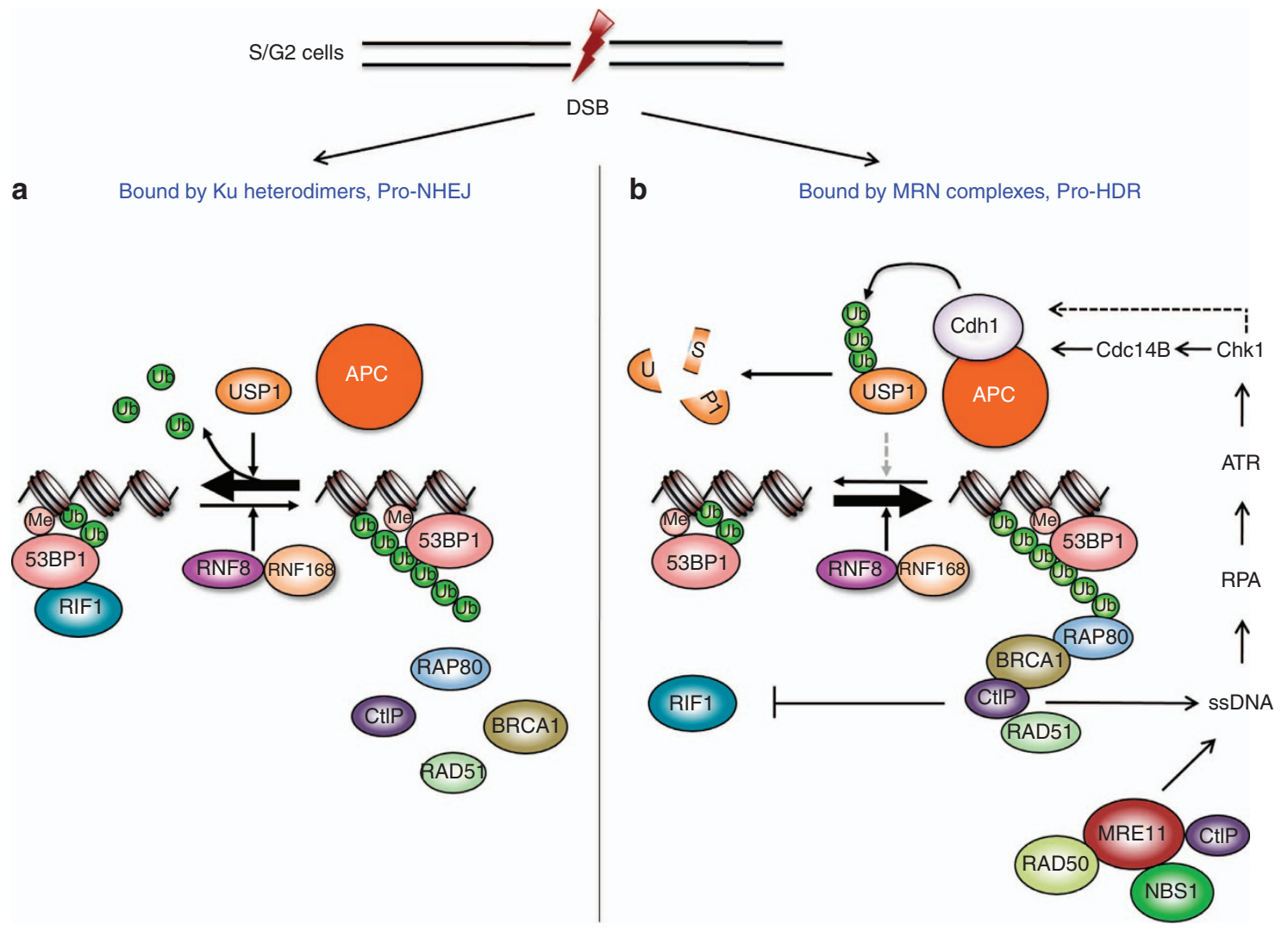

Figure 8 | A model for the regulation of DSB repair pathway choice by USP1 and APC ${ }^{\mathbf{C} d h \mathbf{1}}$ in $\mathbf{S} / \mathbf{G} \mathbf{2}$ cells. The DSBs in S/G2 cells can be processed by two competing factors, the Ku heterodimers and the MRN complex. (a) When a DSB is bound by Ku heterodimers, the break is then destined for NHEJ. (b) On the other hand, when a site is bound by MRN complex, the ends are resected and ssDNA generated, leading to the activation of ATR, Chk1 and APCCAh1, and eventually the destruction of USP1 and the recruitment of BRCA1. BRCA1 then expels RIF1 and reinforces the end resection, leading to further activation of ATR, Chk1 and APC.

(Supplementary Fig. 7d) or a chemical inhibitor (Supplementary Fig. 7e) also strongly inhibited HU-induced BRCA1 focus formation, consistent with Chk1's role in recruiting and activating Cdh1.

\section{Discussion}

We show here that DSBs in S/G2 cells are processed differentially for NHEJ or HDR. Interestingly, the ratio of the number of BRCA1 IRIF over that of RIF1 IRIF varies over a wide range among the cells (Fig. 1a), indicating that the disposition of DSBs between NHEJ and HDR in each cell is different, NHEJ dominates in some but HDR does in some others. It is known that both $\mathrm{Ku}$ heterodimer (for NHEJ) and MRN (MRE11, RAD50 and NBS1) complex (for HDR) can bind to the broken ends upon DNA damage, likely in a competitive fashion ${ }^{57,58}$. We speculate that the stoichiometry of Ku heterodimers and MRN complexes is different in each cell, so the observed differential disposition of DSBs.

While the broken ends are being processed either in favour of NHEJ (bound by $\mathrm{Ku}$ and related factors) or HDR (bound by MRN complex and related factors), ATM- $\gamma \mathrm{H} 2 \mathrm{AX}-\mathrm{MDC} 1$ signalling results in the recruitment of RNF8/RNF168, USP1 and APC among others to the damage sites. RNF168 lays down K63-linked poly-Ubs but only to be destroyed by USP1, so there is no BRCA1 recruitment. However, there must be some remnants of the poly$\mathrm{Ub}$ chains left (for example, see Fig. $2 \mathrm{f}$, there are still some residual $\mathrm{H} 2 \mathrm{AX}-2 \mathrm{Ub}$ ) that are probably enough for 53BP1 but not for BRCA1, as the former is driven to DSB sites primarily by the attraction of methylated histones ${ }^{27,59}$. 53BP1 then brings in RIF1 and the site becomes pro-NHEJ (Fig. 8a). How then is BRCA1 recruited to make or convert those sites bound by MRN complexes for HDR? We showed that the conversion required the function of $\mathrm{APC}^{\mathrm{Cdh} 1}$ and we further demonstrated that the recruitment of Cdh1 depended on Cdc14B and Chk1. Thus, it is likely that MRN complexes along with its associated factors including CtIP bind to the broken ends and initiate end resection, producing ssDNA that activates ATR first then Chk1, which leads to the activation of APC and the destruction of USP1. The latter then allows the accumulation of K63-linked poly-Ub chains and the recruitment of BRCA1. The recruited BRCA1 expels RIF1 on one hand and stimulates end resection further on the other, which reinforces the activation of ATR, Chk1 and APC, making the choice of HDR irreversible (Fig. 8b). In the event of replication stress, the collapsed replication forks already contain ssDNA to begin with which activates ATR and Chk1, committing the damage for HDR. However, APC ${ }^{\mathrm{Cdh} 1}$ is still needed to keep USP1 (and RIF1) out, thus the sensitivity of Cdh1-depleted cells to HU treatment (Fig. 7d).

There have been several reports indicating that Chk1 is essential in homologous recombination repair but the reason is not entirely clear ${ }^{54,60,61}$. We believe that part of the reason is that Chk1 is required to recruit Cdh1 (Fig. 6a). This requirement, at least in part, is mediated by activating $\mathrm{Cdc} 14 \mathrm{~B}$ and bringing the phosphatase to damage sites, although we could not eliminate the possibility that Chk1 might also directly activate Cdh1 at DSB sites (Fig. 8b, dashed arrow).

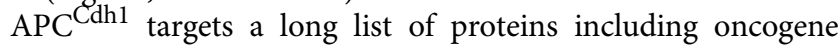
products for degradation. Thus, Cdh1 has been proposed as a 
tumour suppressor ${ }^{62}$. Our data reported clearly show that it also plays an important role in DSB repair, suggesting that its role in the maintenance of genome stability contributes to its function as a potential tumour suppressor.

\section{Methods}

Cell culture and mice. U2OS and U2OS-Fucci cells were cultured in McCoy's 5A medium (Sigma). HEK293T, HeLa, HeLa-Fucci and MEF cells were grown in DMEM (Sigma). All culture media were supplemented with 10\% FBS and 100 units $^{-1}$ penicillin and $100 \mu \mathrm{g} \mathrm{ml}^{-1}$ streptomycin, except the medium for MEFs that was supplemented with 15\% FBS. HEK293T and HeLa cells were obtained from ATCC. U2OS-Fucci cell line was a gift from Dr Hisao Masai (Tokyo Metropolitan Institute of Medical Science) and HeLa-Fucci cell line was a gift from Dr Daniel Durocher (University of Toronto).

The Cdh1 conditional knockout mice were reported previously ${ }^{63}$ and crossed with a transgenic line ubiquitously expressing ER-Cre. Male and female mice at 8 weeks of age were mated and the pregnant females were killed to obtain mouse embryonic fibroblasts (MEFs) as we have done before ${ }^{21,64}$. Homozygous Cdh1 conditional MEFs were infected with GFP- or Cre-expressing adenoviruses to delete Cdh1 for experiments. The mice were in C57/BL6 background. All animal procedures were approved by the IACUC of Baylor College of Medicine.

DNA constructs. All GFP-tagged constructs for human Cdhl, deletion Cdh1 mutants, APC12, RNF8, RNF168, UBE2C, UBE2S, USP1 and OTUB1 were generated by PCR and subcloned into the pEGFP-N1 vector (Clontech). GFP-USP3 construct was a gift from Dr Elisabetta Citterio (Netherlands Cancer Institute). All ubiquitin constructs including WT and UK11 were subcloned into pcDNA3 vector (life technologies) with either 3X FLAG or His tag as indicated. All point and deletion mutations in tumour-associated Cdh1 mutants and in USP1 mutants were introduced by site-directed mutagenesis (Agilent) using pEGFP-N1-Cdh1 or pEGFP-N1-USP1 plasmids as templates. siRNA-resistant Cdh1 and USP1 mutants were also generated by site-directed mutagenesis using pcDNA3-Flag-Cdh1 or pcDNA3-Flag-USP1 plasmids as templates. To generate inducible USP1 expression constructs, USP1 (or its variants) coding region was first subcloned into the pENTRY vector and then transferred to pINDUCER20 via Gateway technology (life technology), using standard methods ${ }^{21}$.

RNA interference. All short interfering RNA (siRNA) duplexes employed in this study were from Sigma. The sequences of siRNAs targeting Cdh1, USP1, BRCA1, RIF1, 53BP1, RNF8, APC3, Chk1, OTUB1, UAF1, Cdc20, CtIP and ATM are as follows: Cdh1 \#1 5'-UGAGAAGUCUCCCAGUCAGdTdT-3', Cdh1 \#2 5'GGAUUAACGAGAAUGAGAAdTdT-3', USP1 \#1 5'-AACCCUAUGUAUGAAGGAUAUdTdT - 3', USP1 \#2 5' - CGGCAAUACUUGCUAUCUUAdTdT$3^{\prime}$, BRCA1 \#1 5'-GGGAUACCAUGCAACAUAAdTdT-3', BRCA1 \#2 5'-CAGCUACCCUUCCAUCAUAdTdT-3', RIF1 \#1 5'-GCATTGACTTCTCACCATAdTdT-3', RIF1 \#2 5'-CGUAGAGAUUAGUGAAACAdTdT-3', 53BP1 5'GAAGGACGGAGUACUAAUAdTdT-3, RNF8 5'-CAGAGAAGCUUACAGAUGUUUdTdT-3', APC3 5'-CAAAAGAGCCUUAGUUUAAdTdT-3', Chk1 \#1 5'GAAGCAGUCGCAGUGAAGAdTdT- $3^{\prime}$, Chk1 \#2 5'-GGGAUAUUAAACCAGAAAAdTdT-3', OTUB1 5'-GCAAGUUCUUCGAGCACUUdTdT-3', USP3 5' GGAGUUAAGGAAUGGGAAAdTdT- ${ }^{\prime}$, UAF1 \#1 5'-GAGAUGAAGUGGAGAAGUdTdT- ${ }^{\prime}$, UAF1 \#2 $5^{\prime}$-CAGCAGAGAUGUAUAGCAAdTdT-3', Cdc20 5' CGAAAUGACUAUUACCUGAdTdT-3', CtIP \#1 5'-GCUAAAACAGGAACGAAUCdTdT-3', CtIP \#2 5' -UCCACAACAUAAUCCUAAUdTdT-3', and ATM 5'-CGCAUGUGAUUAAAGCAACdTdT- $3^{\prime}$. siRNAs targeting MDC1 and Cdc14B were previously described ${ }^{21,65}$. Both plasmid and siRNA transfection were carried out using Lipofectamine 2000 (Life technologies) following the manufacturer's protocol. Cells treated with siRNAs were used for further analyses within $72 \mathrm{~h}$ after transfection.

Antibodies and reagents. The following antibodies were used in this study: antiHis-tag (sc-803, 1:1,000), anti-RNF8 (sc-271462, 1:1,000), anti-APC3 (sc-13154, 1:1,000), anti-BRCA1 (sc-6954, 1:80), anti-cyclin A (sc-751, 1:200), anti-Emi1 (sc-365212, 1:200 (IF), 1:1,000 (WB)), anti-Cdh1 (sc-56312, 1:200 (IF), 1:1,000 (WB)) (Santa Cruz Biotechnology); anti- $\gamma \mathrm{H} 2 \mathrm{AX}$ (05-636, 1:250 (IF), 1:1,000 (WB)), anti-ubiquitin K11 linkage (MABS107-I, 1:200), anti-ubiquitin K63 linkage (05-1308, 1:200 (IF), 1:1,000 (WB)), anti-GAPDH (MAB374, 1:5,000) (Millipore); anti-APC12 (13559-1-AP, 1:100), anti-USP1 (14346-1-AP, 1:200 (IF), 1:1,000 (WB)) (Proteintech); anti-FK2 (BML-PW8810-0100, 1:250) (Enzo Life Sciences); anti-RAP80 (NBP1-87156, 1:250), anti-RAD51 (NB100-148, 1:250), anti-53BP1 (NB100-304, 1:250), anti-DNA-PKcs (phosphoT2609, 1:200) (NB100-2077) (Novus); anti-CtIP (61141, 1:250) (Active Motif); anti-RIF1 (A300-567A, 1:200) (Bethyl Laboratory); anti-MDC1 (ab114143, 1:1,000), anti-H2AX (ab11175, 1:2,000) (Abcam); anti-RPA32 (2208S, 1:250) (Cell Signaling); and anti-FLAG tag (F1804, 1:1,000) (Sigma). KU-55933 was obtained from Santa Cruz Biotechnology. UCN-01 and caffeine were purchased from Sigma.
Western blotting. The cells were collected after treatments and lysed with lysis buffer (50 mM Tris- $\mathrm{HCl}, 150 \mathrm{mM} \mathrm{NaCl}, 1 \%$ Triton X-100 and $0.1 \%$ SDS). SDS loading buffer was added to samples. After boiling, samples were loaded on SDS-PAGE gels and transferred to PVDF membranes. Membranes were probed with indicated antibodies and detection was performed by chemiluminescent immunoassay. Uncropped gel images can be found in Supplementary Fig. 8.

In vivo ubiquitination assay. After transfection of the indicated constructs or siRNAs, HeLa cells stably expressing NLS-BirA and Avi-H2AX were treated with $2 \mu \mathrm{g} \mathrm{ml}^{-1}$ of biotin (Sigma) overnight, and then collected $1 \mathrm{~h}$ after $10 \mathrm{~Gy}$ IR. For endogenous USP1 ubiquitination assay or the interaction between Cdh1 and Emi1, HeLa cells were transfected with the indicated siRNAs and various expression constructs. The transfected cells were synchronized with double thymidine block and released into fresh media for 6-8 $\mathrm{h}$ when they had reached S/G2 (the cell cycle progression was monitored with FACS analysis). In USP1 ubiquitination experiment, MG132 $(10 \mu \mathrm{M})$ was added for $6 \mathrm{~h}$ during the release. The cells were then irradiated with $10 \mathrm{~Gy}$ and collected $1 \mathrm{~h}$ after the irradiation. The collected cells were lysed in $100 \mathrm{mM}$ Tris- $\mathrm{HCl}, \mathrm{pH}$ 8.0, $200 \mathrm{mM} \mathrm{NaCl}, 6 \mathrm{M}$ Urea and $0.5 \%$ SDS (lysis buffer) plus phosphatase (GenDEPOT) and protease inhibitors (Roche) as well as $50 \mu \mathrm{M}$ PR-619 (a pan-DUB inhibitor, Millipore). After sonication, the supernatants were diluted in lysis buffer without SDS and incubated with Streptavidin-Agarose Slurry (Thermo Scientific) or USP1 antibody-conjugated to protein $\mathrm{G}$ beads for $6 \mathrm{~h}$ at $4{ }^{\circ} \mathrm{C}$. The agarose beads were then spun down, washed with the lysis buffer (without SDS), and prepared for Western blot analysis.

Quantitative RT-PCR. Total RNA was extracted using RNeasy mini kit (Qiagen) and CDNA was prepared using RT reagent kit (Takara). quantitative PCR with reverse transcription was performed on a StepOne Plus Real-Time PCR system (Applied Biosystems) using FastStart Universal SYBR Green master mix (Roche) Expression of GAPDH was used to normalize relative expression of Cdc14b mRNA.

Laser microirradiation. The cells were plated on $35 \mathrm{~mm}$ glass bottom dishes (MatTek Corporation) overnight. Twenty-four hours after transfection with the indicated constructs, the cells were treated with $1 \mu \mathrm{g} \mathrm{ml}^{-1}$ of Hoechst 33258 (Sigma) for $2 \mathrm{~h}$ and DNA damages were introduced by micro-irradiation using Nikon A1R-s inverted confocal microscope (Nikon, Inc.) equipped with a $405 \mathrm{~nm}$ diode laser focused through a $\times 60$ Plan Apo/1.4 NA Oil objective and a $37^{\circ} \mathrm{C}$ heating chamber. Time-lapse imaging for the recruitment of EGFP- and RFPtagged proteins to sites of DNA damage was recorded after excitation with 488 and $561 \mathrm{~nm}$ lasers, respectively. For the recruitment of fluorescence-tagged proteins in the presence of chemical inhibitors, cells were treated with indicated inhibitors for $24 \mathrm{~h}$ on the second day of the transfection. For live cell imaging in siRNAtreated cells, the indicated constructs were transfected $48 \mathrm{~h}$ after the siRNA transfection.

HDR and NHEJ assays. Briefly, U2OS cells carrying the HR repair reporter DRGFP were transfected with pCBA-I-SceI or pCAGGS (mock transfection) $48 \mathrm{~h}$ after the transfection of the indicated siRNAs. pCAGGS-mCherry was co-transfected as a control for transfection efficiency. Forty-eight hours later, flow cytometry analysis was performed to detect GFP and mCherry-positive cells using LSR Fortessa (BD Biosciences). For NHEJ assay, U2OS cells were transfected with the indicated siRNAs for $72 \mathrm{~h}$, and then cells were electroporated with $5 \mu \mathrm{g}$ of linearized pCSCMV:tdTomato and $5 \mu \mathrm{g}$ of pEGFP-N1 per $10^{6}$ cells. After $5 \mathrm{~h}$ culture, the cells were collected for flow cytometry analysis. The data were analysed with $\mathrm{BD}$ FACSDiva software.

Immunofluorescence microscopy. The cells were cultured on coverslips overnight, and transfected with indicated siRNAs. Seventy-two hours after transfection, the cells were treated with gamma irradiation $(2 \mathrm{~Gy})$ and collected to visualize the IRIF. For pre-extraction, the cells were washed with PBS and incubated with preextraction buffer $(0.2 \%$ Triton X-100 and $300 \mathrm{mM}$ sucrose) for $2 \mathrm{~min}$ on ice. Then, the cells were fixed with $4 \%$ paraformaldehyde for $15 \mathrm{~min}$ at room temperature, and permeabilized with $0.5 \%$ Triton X-100 for $15 \mathrm{~min}$. Samples were blocked with $5 \%$ goat serum and incubated with primary antibodies for $1 \mathrm{~h}$ at $37^{\circ} \mathrm{C}$. After washing with PBS three times, the samples were incubated with secondary antibodies for $1 \mathrm{~h}$ at $37^{\circ} \mathrm{C}$. DNA was visualized with DAPI. Images were acquired on Deltavision LIVE high-resolution deconvolution microscope (Applied Biosystems) For the quantification of focus number and intensity, deconvoluted images were analysed using softWoRx Explorer (Applied Precisions), CellProfiler (Broad Institute) and Prism 6 (GraphPad). The profile plots of fluorescence intensities were generated using ImageJ. $P$ values were calculated with a student's $t$-test by Prism 6 and data were considered statistically significant for $P$ values $<0.05$.

Cell viability assay. U2OS cells were transfected with the indicated siRNAs for $48 \mathrm{~h}$, split into a 96 -well plate, and treated with different concentrations of 
hydroxyurea for $24 \mathrm{~h}$. After washing with PBS, the cells were cultured in fresh media for additional 2 days, and incubated with $0.5 \mathrm{mg} \mathrm{ml}^{-1}$ MTT for $4 \mathrm{~h}$. A solubilization solution $(10 \% \mathrm{SDS} / 0.01 \mathrm{M} \mathrm{HCl})$ was added into each well and the absorbance at $595 \mathrm{~nm}$ of the samples was measured using a microplate reader.

Data availability. All relevant data are available from the authors.

\section{References}

1. Jackson, S. P. \& Bartek, J. The DNA-damage response in human biology and disease. Nature 461, 1071-1078 (2009).

2. Lieber, M. R. The mechanism of double-strand DNA break repair by the nonhomologous DNA end-joining pathway. Annu. rev. biochem. 79, 181-211 (2010).

3. Symington, L. S. \& Gautier, J. Double-strand break end resection and repair pathway choice. Annu. rev. genet. 45, 247-271 (2011).

4. Chapman, J. R., Taylor, M. R. \& Boulton, S. J. Playing the end game: DNA double-strand break repair pathway choice. Mol. cell 47, 497-510 (2012).

5. Li, M., York, J. P. \& Zhang, P. Loss of Cdc20 causes a securin-dependent metaphase arrest in two-cell mouse embryos. Mol. cell. biol. 27, 3481-3488 (2007).

6. Jin, L., Williamson, A., Banerjee, S., Philipp, I. \& Rape, M. Mechanism of ubiquitin-chain formation by the human anaphase-promoting complex. Cell 133, 653-665 (2008).

7. Bu, W., Xin, L., Toneff, M., Li, L. \& Li, Y. Lentivirus vectors for stably introducing genes into mammary epithelial cells in vivo. J. Mammary Gland Biol. Neoplasia 14, 401-404 (2009).

8. Cotto-Rios, X. M., Jones, M. J., Busino, L., Pagano, M. \& Huang, T. T. APC/CCdh1-dependent proteolysis of USP1 regulates the response to UVmediated DNA damage. J. cell biol. 194, 177-186 (2011).

9. Zhang, L. et al. Proteolysis of Rad17 by Cdh1/APC regulates checkpoint termination and recovery from genotoxic stress. $E M B O j .29,1726-1737$ (2010).

10. Bassermann, F. et al. The Cdc14B-Cdh1-Plk1 axis controls the G2 DNAdamage-response checkpoint. Cell 134, 256-267 (2008).

11. Lafranchi, L. et al. APC/C(Cdh1) controls CtIP stability during the cell cycle and in response to DNA damage. EMBO j. 33, 2860-2879 (2014).

12. Kramer, E. R., Scheuringer, N., Podtelejnikov, A. V., Mann, M. \& Peters, J. M. Mitotic regulation of the APC activator proteins $\mathrm{CDC} 20$ and $\mathrm{CDH1}$. Mol. biol. cell 11, 1555-1569 (2000).

13. Zhou, Y., Ching, Y. P., Chun, A. C. \& Jin, D. Y. Nuclear localization of the cell cycle regulator $\mathrm{CDH1}$ and its regulation by phosphorylation. J. Biol. Chem. 278, 12530-12536 (2003)

14. Visintin, R. et al. The phosphatase Cdc14 triggers mitotic exit by reversal of Cdk-dependent phosphorylation. Mol. Cell 2, 709-718 (1998).

15. Stegmeier, F. \& Amon, A. Closing mitosis: the functions of the Cdc14 phosphatase and its regulation. Annu. rev. genet. 38, 203-232 (2004).

16. Kaiser, B. K., Zimmerman, Z. A., Charbonneau, H. \& Jackson, P. K. Disruption of centrosome structure, chromosome segregation, and cytokinesis by misexpression of human Cdc14A phosphatase. Mol. Biol. Cell 13, 2289-2300 (2002).

17. Mailand, N. et al. Deregulated human Cdc14A phosphatase disrupts centrosome separation and chromosome segregation. Nat. Cell Biol. 4, 317-322 (2002).

18. Mocciaro, A. et al. Vertebrate cells genetically deficient for Cdc14A or Cdc14B retain DNA damage checkpoint proficiency but are impaired in DNA repair J. cell biol. 189, 631-639 (2010).

19. Berdougo, E., Nachury, M. V., Jackson, P. K. \& Jallepalli, P. V. The nucleolar phosphatase Cdc14B is dispensable for chromosome segregation and mitotic exit in human cells. Cell Cycle 7, 1184-1190 (2008).

20. Schmitz, M. H. et al. Live-cell imaging RNAi screen identifies PP2A-B55alpha and importin-betal as key mitotic exit regulators in human cells. Nat. cell biol. 12, 886-893 (2010).

21. Lin, H. et al. Cdc14A and Cdc14B redundantly regulate DNA double-strand break repair. Mol. cell. biol. 35, 3657-3668 (2015).

22. Peddibhotla, S. et al. The DNA damage effector Chk1 kinase regulates Cdc14B nucleolar shuttling during cell cycle progression. Cell cycle 10, 671-679 (2011).

23. Zhao, Y., Brickner, J. R., Majid, M. C. \& Mosammaparast, N. Crosstalk between ubiquitin and other post-translational modifications on chromatin during double-strand break repair. Trends Cell Biol. 24, 426-434 (2014).

24. Polo, S. E. \& Jackson, S. P. Dynamics of DNA damage response proteins at DNA breaks: a focus on protein modifications. Genes dev. 25, 409-433 (2011).

25. Mattiroli, F. et al. RNF168 ubiquitinates K13-15 on H2A/H2AX to drive DNA damage signaling. Cell 150, 1182-1195 (2012).

26. Acs, K. et al. The AAA-ATPase VCP/p97 promotes 53BP1 recruitment by removing L3MBTL1 from DNA double-strand breaks. Nat. Struct. Mol. Biol. 18, 1345-1350 (2011).

27. Fradet-Turcotte, A. et al. 53BP1 is a reader of the DNA-damage-induced $\mathrm{H} 2 \mathrm{~A}$ Lys 15 ubiquitin mark. Nature 499, 50-54 (2013).
28. Powell, S. N. \& Kachnic, L. A. Roles of BRCA1 and BRCA2 in homologous recombination, DNA replication fidelity and the cellular response to ionizing radiation. Oncogene 22, 5784-5791 (2003).

29. Scully, R. et al. Dynamic changes of BRCA1 subnuclear location and phosphorylation state are initiated by DNA damage. Cell 90, 425-435 (1997).

30. Chapman, J. R. et al. RIF1 is essential for 53BP1-dependent nonhomologous end joining and suppression of DNA double-strand break resection. Mol. cell 49, 858-871 (2013).

31. Escribano-Diaz, C. et al. A cell cycle-dependent regulatory circuit composed of 53BP1-RIF1 and BRCA1-CtIP controls DNA repair pathway choice. Mol. cell 49, 872-883 (2013)

32. Zimmermann, M., Lottersberger, F., Buonomo, S. B., Sfeir, A. \& de Lange, T. 53BP1 regulates DSB repair using Rif1 to control $5^{\prime}$ end resection. Science 339, 700-704 (2013).

33. Zhang, H. et al. A cell cycle-dependent BRCA1-UHRF1 cascade regulates DNA double-strand break repair pathway choice. Nat. commun. 7, 10201 (2016).

34. Mao, Z., Bozzella, M., Seluanov, A. \& Gorbunova, V. DNA repair by nonhomologous end joining and homologous recombination during cell cycle in human cells. Cell cycle 7, 2902-2906 (2008).

35. Branzei, D. \& Foiani, M. Regulation of DNA repair throughout the cell cycle. Nat. rev. Mol. cell biol. 9, 297-308 (2008).

36. Daley, J. M. \& Sung, P. 53BP1, BRCA1, and the choice between recombination and end joining at DNA double-strand breaks. Mol. cell. biol. 34, 1380-1388 (2014).

37. Kakarougkas, A. \& Jeggo, P. A. DNA DSB repair pathway choice: an orchestrated handover mechanism. Br. J. Radiol. 87, 20130685 (2014)

38. Raderschall, E., Golub, E. I. \& Haaf, T. Nuclear foci of mammalian recombination proteins are located at single-stranded DNA regions formed after DNA damage. Proc. Natl Acad. Sci. USA 96, 1921-1926 (1999).

39. Zou, L. \& Elledge, S. J. Sensing DNA damage through ATRIP recognition of RPA-ssDNA complexes. Science 300, 1542-1548 (2003).

40. Nakada, S. et al. Non-canonical inhibition of DNA damage-dependent ubiquitination by OTUB1. Nature 466, 941-946 (2010).

41. Sharma, N. et al. USP3 counteracts RNF168 via deubiquitinating H2A and gammaH2AX at lysine 13 and 15. Cell cycle 13, 106-114 (2014).

42. Mosbech, A., Lukas, C., Bekker-Jensen, S. \& Mailand, N. The deubiquitylating enzyme USP44 counteracts the DNA double-strand break response mediated by the RNF8 and RNF168 ubiquitin ligases. J. Biol. Chem. 288, 16579-16587 (2013).

43. Morris, J. R. Attenuation of the ubiquitin conjugate DNA damage signal by the proteasomal DUB POH1. Cell Cycle 11, 4103-4104 (2012).

44. Nicassio, F. et al. Human USP3 is a chromatin modifier required for S phase progression and genome stability. Curr. biol. 17, 1972-1977 (2007).

45. Thorslund, T. et al. Histone $\mathrm{H} 1$ couples initiation and amplification of ubiquitin signalling after DNA damage. Nature 527, 389-393 (2015).

46. Cohn, M. A. et al. A UAF1-containing multisubunit protein complex regulates the Fanconi anemia pathway. Mol. cell 28, 786-797 (2007).

47. Coster, G. et al. The DNA damage response mediator MDC1 directly interacts with the anaphase-promoting complex/cyclosome. J. biol. chem. 282, 3205332064 (2007).

48. Miller, J. J. et al. Emil stably binds and inhibits the anaphase-promoting complex/cyclosome as a pseudosubstrate inhibitor. Genes dev. 20, 2410-2420 (2006).

49. Sudo, T. et al. Activation of Cdh1-dependent APC is required for G1 cell cycle arrest and DNA damage-induced G2 checkpoint in vertebrate cells. EMBO J. 20, 6499-6508 (2001).

50. Wiebusch, L. \& Hagemeier, C. p53- and p21-dependent premature APC/C-Cdh1 activation in G2 is part of the long-term response to genotoxic stress. Oncogene 29, 3477-3489 (2010).

51. Shimada, M. et al. Chk1 is a histone H3 threonine 11 kinase that regulates DNA damage-induced transcriptional repression. Cell 132, 221-232 (2008).

52. Smits, V. A., Reaper, P. M. \& Jackson, S. P. Rapid PIKK-dependent release of Chk1 from chromatin promotes the DNA-damage checkpoint response. Curr. biol. 16, 150-159 (2006).

53. Mimitou, E. P. \& Symington, L. S. DNA end resection: many nucleases make light work. DNA Repair (Amst) 8, 983-995 (2009).

54. Sorensen, C. S. et al. The cell-cycle checkpoint kinase Chk1 is required for mammalian homologous recombination repair. Nat. Cell Biol. 7, 195-201 (2005).

55. Arnaudeau, C., Lundin, C. \& Helleday, T. DNA double-strand breaks associated with replication forks are predominantly repaired by homologous recombination involving an exchange mechanism in mammalian cells J. mol. biol. 307, 1235-1245 (2001).

56. Gaillard, H., Garcia-Muse, T. \& Aguilera, A. Replication stress and cancer. Nature rev. Cancer 15, 276-289 (2015).

57. Sun, J., Lee, K. J., Davis, A. J. \& Chen, D. J. Human Ku70/80 protein blocks exonuclease 1-mediated DNA resection in the presence of human Mrel1 or Mre11/Rad50 protein complex. J. Biol. Chem. 287, 4936-4945 (2012). 
58. Hartlerode, A. J., Morgan, M. J., Wu, Y., Buis, J. \& Ferguson, D. O. Recruitment and activation of the ATM kinase in the absence of DNA-damage sensors. Nat. Struct. Mol. Biol. 22, 736-743 (2015).

59. Mallette, F. A. et al. RNF8- and RNF168-dependent degradation of KDM4A/ JMJD2A triggers 53BP1 recruitment to DNA damage sites. EMBO J. 31, 1865-1878 (2012)

60. Hu, B. et al. Fhit and CHK1 have opposing effects on homologous recombination repair. Cancer Res. 65, 8613-8616 (2005).

61. Wang, H., Hu, B., Liu, R. \& Wang, Y. CHK1 affecting cell radiosensitivity is independent of non-homologous end joining. Cell Cycle 4, 300-303 (2005).

62. Li, M. \& Zhang, P. The function of APC/CCdh1 in cell cycle and beyond. Cell div. 4, 2 (2009).

63. Garcia-Higuera, I. et al. Genomic stability and tumour suppression by the APC/C cofactor Cdh1. Nat. Cell Biol. 10, 802-811 (2008).

64. Wei, Z. et al. Early-onset aging and defective DNA damage response in Cdc14b-deficient mice. Mol. cell. biol. 31, 1470-1477 (2011).

65. Galanty, Y. et al. Mammalian SUMO E3-ligases PIAS1 and PIAS4 promote responses to DNA double-strand breaks. Nature 462, 935-939 (2009).

\section{Acknowledgements}

We thank S.-Y. Lin (MD Anderson Cancer Center) for cell lines; J. Rosen (Baylor College of Medicine) for reagents; H. Masai (Tokyo Metropolitan Institute of Medical Science) for U2OS-Fucci cell line; D. Durocher (University of Toronto) for HeLa-Fucci cell line; E. Citterio (Netherlands Cancer Institute) for GFP-USP3 construct; M.S.Y. Huen (The University of Hong Kong) for RNF168 antibody. This work was performed with facilities and instruments in the Imaging Core of National Center for Protein Science (Beijing), the Cytometry and Cell Sorting Core at Baylor College of Medicine with funding from the NIH (P30 AI036211, P30 CA125123 and S10 RR024574), the Integrated Microscopy Core at Baylor College of Medicine with funding from the NIH (HD007495, DK56338 and CA125123), and the John S. Dunn Gulf Coast Consortium for Chemical Genomics. We also thank other members of the Zhang lab for helpful discussion and support.

This work was supported in part by an international collaboration grant

(\# 2013DFB30210) and a 973 Project grant (\# 2013CB910300) from Chinese Minister of Science and Technology, in part by a Chinese National Natural Science Foundation grant (\# 81171920), in part by a grant from The Committee of Science and Technology of Beijing Municipality, China (\# Z141100000214015), and in part by NIH grants CA116097 and CA122623 to P.Z. J.J. is supported by grants from National Institutes of Health (R01GM102529) and the Welch Foundation (AU-1711). S.H. is supported by grants (\# 81272488 and 81472795) from Chinese National Natural Science Foundation. Y.Z. is supported by grants from the National Natural Scientific Foundation of China
(No. 81430055), Programs for Changjiang Scholars and Innovative Research Team in University (No. IRT_15R13).

\section{Author contributions}

K.H., C.M., H.L., L.T., Z.L., F.Z. and J.-M.L. designed and performed experiments and analysed the results. B.Z., H.P., S.H., M.M., J.J., H.C., Y.Z., Q.Z. and P.Z. analysed and discussed the results, and wrote the manuscript.

\section{Additional information}

Supplementary Information accompanies this paper at http://www.nature.com/ naturecommunications

Competing interests: The authors declare no competing financial interests.

Reprints and permission information is available online at http://npg.nature.com/ reprintsandpermissions/

How to cite this article: Ha, K. et al. The anaphase promoting complex impacts repair choice by protecting ubiquitin signalling at DNA damage sites. Nat. Commun. 8, 15751 doi: 10.1038/ncomms15751 (2017).

Publisher's note: Springer Nature remains neutral with regard to jurisdictional claims in published maps and institutional affiliations.

cC Open Access This article is licensed under a Creative Commons Attribution 4.0 International License, which permits use, sharing, adaptation, distribution and reproduction in any medium or format, as long as you give appropriate credit to the original author(s) and the source, provide a link to the Creative Commons license, and indicate if changes were made. The images or other third party material in this article are included in the article's Creative Commons license, unless indicated otherwise in a credit line to the material. If material is not included in the article's Creative Commons license and your intended use is not permitted by statutory regulation or exceeds the permitted use, you will need to obtain permission directly from the copyright holder. To view a copy of this license, visit http://creativecommons.org/ licenses/by/4.0/

(C) The Author(s) 2017 


\section{Erratum: The anaphase promoting complex impacts repair choice by protecting ubiquitin signalling at DNA damage sites}

Kyungsoo Ha, Chengxian Ma, Han Lin, Lichun Tang, Zhusheng Lian, Fang Zhao, Ju-Mei Li, Bei Zhen, Huadong Pei, Suxia Han, Marcos Malumbres, Jianping Jin, Huan Chen, Yongxiang Zhao, Qing Zhu \& Pumin Zhang

Nature Communications 8:15751 doi: 10.1038/ncomms15751 (2017); Published 12 Jun 2017; Updated 29 Nov 2017.

This Article contains an error in Fig. 7. In Fig. 7b, the label 'siBRCRA1' should have read 'si53BP1'. The correct version of the figure appears below as Fig. 1 .

(c) Open Access This article is licensed under a Creative Commons Attribution 4.0 International License, which permits use, sharing, adaptation, distribution and reproduction in any medium or format, as long as you give appropriate credit to the original author(s) and the source, provide a link to the Creative Commons license, and indicate if changes were made. The images or other third party material in this article are included in the article's Creative Commons license, unless indicated otherwise in a credit line to the material. If material is not included in the article's Creative Commons license and your intended use is not permitted by statutory regulation or exceeds the permitted use, you will need to obtain permission directly from the copyright holder. To view a copy of this license, visit http://creativecommons.org/licenses/by/4.0/

(C) The Author(s) 2017 


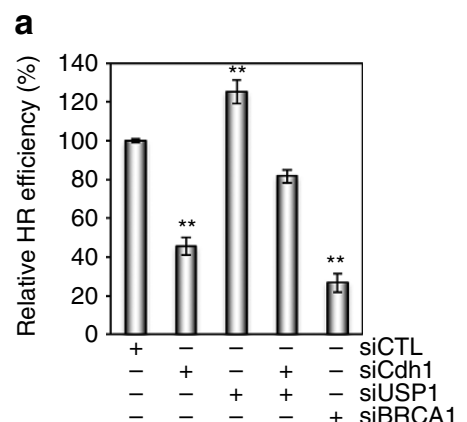

d

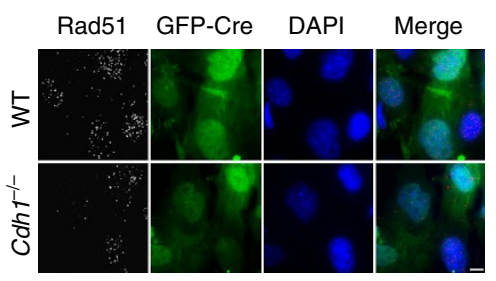

\section{b}

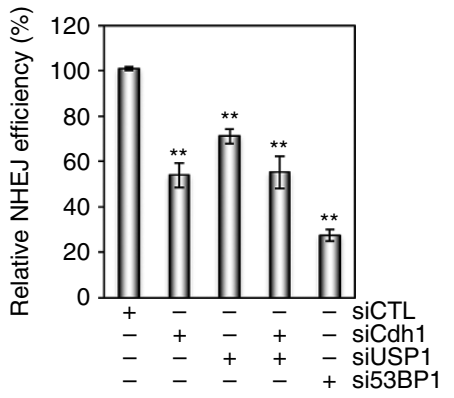

c

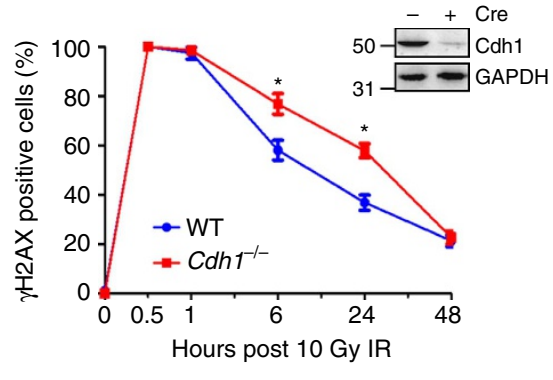

e

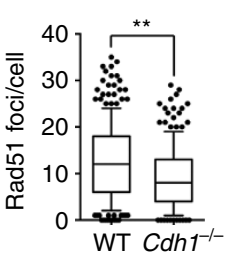

e

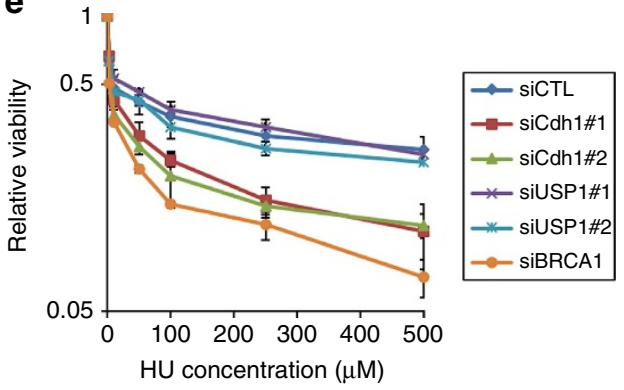

f
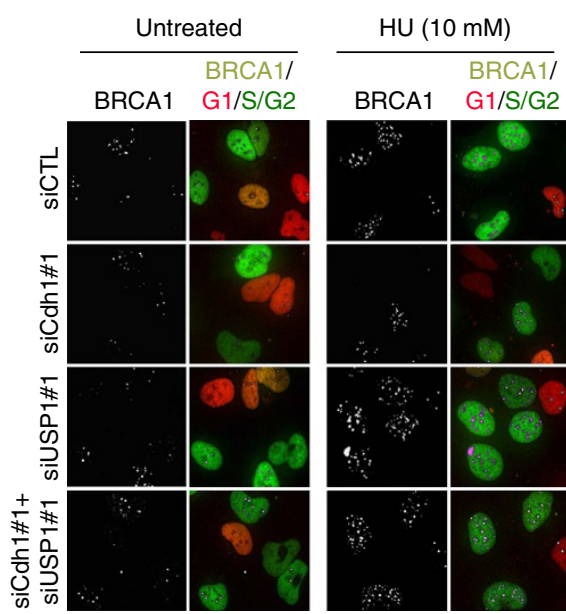

g
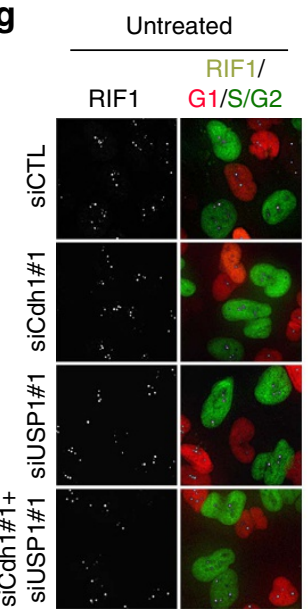

$\frac{\mathrm{HU}(10 \mathrm{mM})}{\mathrm{RIF} 1 /}$
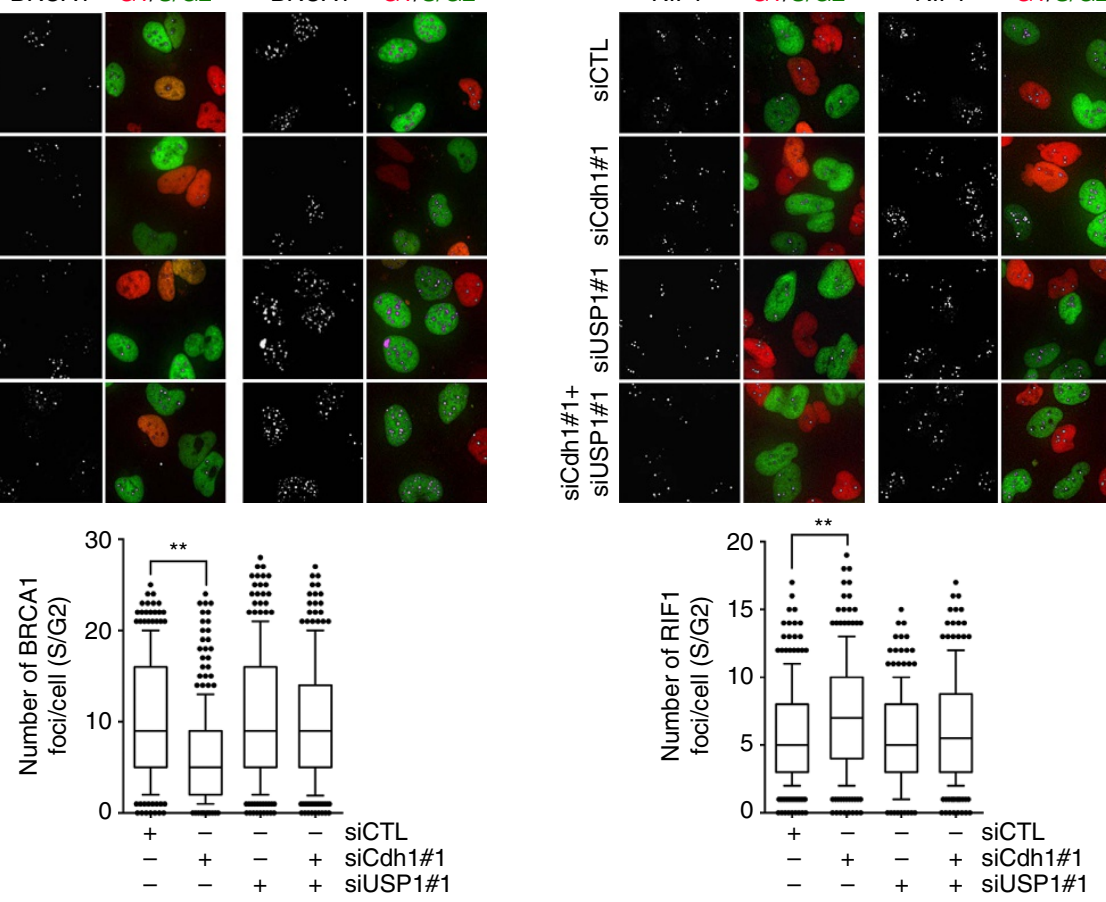

Figure 1 